Notre Dame Journal of Formal Logic

Volume 37, Number 2, Spring 1996

\title{
Decidability Results for Metric and Layered Temporal Logics
}

\author{
ANGELO MONTANARI and ALBERTO POLICRITI
}

\begin{abstract}
We study the decidability problem for metric and layered temporal logics. The logics we consider are suitable to model time granularity in various contexts, and they allow one to build granular temporal models by referring to the "natural scale" in any component of the model and by properly constraining the interactions between differently-grained components. A monadic second-order language combining operators such as temporal contextualization and projection, together with the usual displacement operator of metric temporal logics, is considered, and the theory of finitely-layered metric temporal structures is shown to be decidable.
\end{abstract}

1 Introduction Temporal logic has been used widely and successfully to model and reason about temporal knowledge in several fields of computer science, including software engineering, database, and artificial intelligence. Almost all of the proposed logics of time assume a flat temporal model, constraining temporal information to be specified at a single level of granularity. As noticed in van Benthem [19], being able to provide and relate temporal representations at different "grain levels" of the same reality is an important research theme for temporal logic and a major requirement for many applications.

With regard to logical specifications, there exists a large class of real-time systems whose components have dynamic behavior regulated by very different time constants (granular systems). A good specification language must enable one to specify and verify the components of a granular system and their interactions in a simple and intuitively clear way, see e.g., Fiadeiro and Maibaum [8]. With regard to temporal databases, when information is collected from different sources which are not under the same control, differently-grained time-stamps are associated with different data. To guarantee consistency either the data must be converted into a uniform representation that is independent of time-granularity, or temporal operations must be generalized to cope with data associated with different temporal domains. In both cases, a precise semantics for time granularity is needed; see e.g., Wang et al. [20]. With regard to problem solving, intelligent temporal reasoning systems should be able to 
switch among time granularities in order to provide either quick coarse-grain answers or slower fine-grain ones, depending on the requirements for responsiveness and quality of the answer, see e.g., Euzenat [7]. Finally, shifts in the temporal perspective occur very often in natural language communication, and thus the ability to support and relate a variety of temporal models, at different grain sizes, is a relevant feature for the task of natural language understanding. For all of these application domains (and many others), the flatness of the temporal model underlying most logics of time proposed in the literature is a major drawback.

In 4 and 13], Montanari et al. proposed a metric and layered temporal logic (MLTL for short) for specifying granular real-time systems. Metric temporal logics, e.g., Koymans [11], and Montanari and de Rijke [14], extend propositional logic with a parameterized operator of relative temporal realization. MLTL can be viewed as the combination of a number of differently-grained metric temporal logics. It replaces the flat temporal domain of metric temporal logics with a temporal universe consisting of a set of differently-grained temporal domains together with relations between instants belonging to different domains. To qualify formulas with respect to the temporal universe, MLTL is equipped with an operator of contextualization that identifies the domains a given formula refers to. Within each temporal domain, it is then possible to talk about truth and falsehood of formulas at different time instants by means of a displacement operator. Finally, a projection operator can be used to constrain the relationships between formulas associated with differently-grained domains.

The combined use of these operators allows one to represent a granular system by properly connecting a set of differently-grained formulas. In the simplest case, this might be just a boolean combination of formulas referring to different temporal domains. In more complex cases, the projection operator is used to deal with nested quantifications of differently-grained temporal displacements (e.g., to specify the condition: "there exist some days during which the plant remains inactive for some hours"), or to specify the composition of differently-grained temporal displacements (e.g., to specify the condition: "in twenty seconds, five minutes will have passed from the occurrence of the fault"). Moreover, MLTL can be provided with consistency rules that, given the truth value of a formula with respect to the domains it explicitly refers to, constrain its truth value with respect to other domains (a detailed discussion can be found in [4]).

This paper focuses on the decidability of the validity and satisfiability problems for MLTL. In [1], Alur and Henzinger showed that, under suitable assumptions about the temporal domain and the associated operations, the validity and satisfiability problems for real-time logics are decidable. These problems can be reduced, through coding into the theory $\mathbf{S 1 S}$, to the decidable problem of determining whether or not the language recognized by a given Büchi automaton is empty (Thomas 18$]$ ). ${ }^{1}$ Our goal is to generalize this result to temporal logics combining metric and layered features.

When faced with a combined logic, there are at least two possible approaches to the problem of establishing its logical properties such as decidability, soundness, and completeness. The first identifies what constraints the combination method must satisfy to guarantee the transfer of logical properties from the component logics to the combined one; examples can be found in Finger and Gabbay 9 and Montanari and de Rijke [14]. Otherwise, instead of lifting logical properties from the components to 
the combined logic, one can try to obtain a reduction to one of components and solve the problem for that one component. In this paper we follow the latter strategy by embedding finitely-layered metric temporal structures into their finest metric component, and then reducing the decidability of the theory of the simplest component to a theory that is known to be decidable, namely S1S; cf. Ershov et al. in 6] and Rabin 17 .

The paper is organized as follows. In Section 2, we introduce the theory of finitely-layered metric temporal structures. In Section 3 . we show how to reduce the decidability problem for this theory to the decidability problem for S1S. Conclusions provide an assessment of the work and outline the current topics of research.

2 The theory of finitely-layered metric temporal structures Let $\mathcal{L}_{L M}^{2}$ be the second-order language for the theory of finitely-layered metric temporal structures $T_{L M}$. It includes individual variables $\overrightarrow{\mathrm{x}}, \overrightarrow{\mathrm{y}}, \ldots$ and uninterpreted unary predicate symbols, the constant symbol $\overrightarrow{0}$, the unary function symbols $\overrightarrow{+}_{1} 1, \ldots, \overrightarrow{+}_{n} 1$ (local successors), the unary (interpreted) predicate symbols $\overrightarrow{\mathrm{T}}^{1}, \ldots, \overrightarrow{\mathrm{T}}^{\mathrm{n}}$ (contextualizations), the binary relational symbols $\overrightarrow{\leq}_{1}, \ldots, \overrightarrow{\leq}_{\mathrm{n}}$ (local orderings), $\uparrow$ (upward projection) and $\downarrow$ (downward projection), $\vec{\equiv}_{1,2}, \vec{\equiv}_{1,3}, \ldots, \vec{\equiv}_{n, 2}, \vec{\equiv}_{n, 3}, \ldots$ (local congruences), and quantification of individual variables and (uninterpreted) unary predicate symbols. The first-order fragment of $\mathcal{L}_{L M}^{2}$ is denoted by $\mathcal{L}_{L M}$. We restrict ourselves to formulas that contain no free individual variables. Setting up the structures in which $\mathcal{L}_{L M}^{2}$ can be interpreted is our next task; it takes quite a bit of work.

We define a finitely-layered metric temporal structure as a tuple

$$
\begin{aligned}
\left(\overrightarrow{\mathcal{T}}, \vec{T}^{1}, \ldots, \vec{T}^{n},\right. & \overrightarrow{\leq}_{1}, \ldots, \overrightarrow{\leq}_{n}, \uparrow, \downarrow, \vec{\equiv}_{1,2}, \vec{\equiv}_{1,3}, \ldots, \\
& \left.\vec{\equiv}_{n, 2}, \vec{\equiv}_{n, 3}, \ldots, \overrightarrow{+}_{1} 1, \ldots, \overrightarrow{+}_{n} 1, \overrightarrow{0}\right) .
\end{aligned}
$$

$\overrightarrow{\mathcal{T}}$ is the carrier set of the structure, and it is called the temporal universe. The $n$ components $\vec{T}^{1}, \ldots, \vec{T}^{n}$ are sets of temporal vectors corresponding to the interpretation of the $n$ unary predicates $\overrightarrow{\mathrm{T}}^{1}, \ldots, \overrightarrow{\mathrm{T}}^{\mathrm{n}}$, respectively. The temporal universe $\overrightarrow{\mathcal{T}}$ is equal to $\bigcup_{i=1}^{n} \vec{T}^{i}$. The set of domains is totally ordered by inclusion: $\vec{T}^{1} \supset \vec{T}^{2} \supset \cdots \supset \vec{T}^{n}$, and thus $\overrightarrow{\mathcal{T}}=\vec{T}^{1}$. Let us call $\supset$ the granularity relation. For each pair of domains $\vec{T}^{i}, \vec{T}^{j}$, we say that the granularity of $\vec{T}^{i}$ is coarser (resp. finer) than the granularity of $\vec{T}^{j}$ if and only if $\vec{T}^{i} \supset \vec{T}^{j}$ (resp. $\left.\vec{T}^{j} \supset \vec{T}^{i}\right)$. Formally, a granularity relation on $\left\{\vec{T}^{1}, \ldots, \vec{T}^{n}\right\}$ is a total ordering $\supset$ such that $\vec{T}^{i} \supset \vec{T}^{j}$, for $1 \leq i<n-1$ and $i<j \leq n$. Each vector $\vec{x}$ such that $\vec{T}^{i}$ is the finest domain to which it belongs is called a time instant of $\vec{T}^{i}$. A fine membership relation $\epsilon^{\prime}$ is defined such that $\vec{x} \in^{\prime} \vec{T}^{i}$ if and only if $\vec{x} \in \vec{T}^{i} \wedge \vec{x} \notin \vec{T}^{i+1}$. Since $n$ is finite, for each $\vec{x} \in \overrightarrow{\mathcal{T}}$, there exists one and only one $\vec{T}^{i}$ such that $\vec{x} \in \vec{T}^{i}$. Moreover, for each pair of consecutive domains $\vec{T}^{i}, \vec{T}^{i+1}$, with $1 \leq i<n$, we assume that there exists a natural number $c f_{i, i+1}$, called the conversion factor between $\vec{T}^{i}$ and $\vec{T}^{i+1}$, that expresses the ratio between the granularities of time instants finely belonging to the two domains (homogeneity assumption).

Furthermore, $\overrightarrow{\leq}_{1}, \ldots, \overrightarrow{\leq}_{n}$ are binary relations of local temporal ordering over $\vec{T}^{1}, \ldots, \vec{T}^{n}$, respectively; $\uparrow$ and $\downarrow$ are binary relations of upward and downward pro-

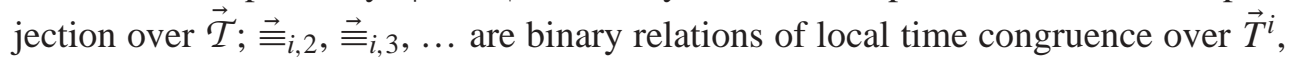


for $1 \leq i \leq n ; \overrightarrow{+}_{1} 1, \ldots, \overrightarrow{+}_{n} 1$ are unary successor functions of temporal displacement over $\vec{T}^{1}, \ldots, \vec{T}^{n}$, respectively; and $\overrightarrow{0}$ is the zero vector (see below).

To specify the components of finitely-layered metric temporal structures, we introduce a representation for temporal vectors. For $1 \leq i \leq n$, we represent the set $\left\{\vec{x} \mid \vec{x} \in \vec{T}^{i}\right\}$ as the generalized cartesian product $\mathbb{N} \times \prod_{k=1}^{i-1}\left[0, c f_{k, k+1}\right)$, where each pair $\left[0, c f_{k, l}\right)$ denotes an interval of natural numbers. The representation of the $\operatorname{set}\{\vec{x} \mid$ $\left.\vec{x} \in \vec{T}^{i}\right\}$ is thus simply $\bigcup_{j=i}^{n} \mathbb{N} \times \prod_{k=1}^{j-1} \times\left[0, c f_{k, k+1}\right)$. Furthermore, for $k=1, \ldots, n$, a function $[\cdot]_{k}: \overrightarrow{\mathcal{T}} \mapsto \mathbb{N} \cup\{\perp\}$ can be defined such that, for each $\vec{x}\left(\in^{\prime} T^{i}\right),[\vec{x}]_{k}$ is equal to the $k$-th component of $\vec{x}$ if $k \leq i$, and to $\perp$ otherwise.

The above representation of temporal vectors can be interpreted as follows. Time instants finely belonging to $\vec{T}^{1}$ take value over (a temporal domain isomorphic to) $\mathbb{N}$. Let us call their values absolute temporal positions. The representation of an instant $\vec{x}$ finely belonging to $\vec{T}^{i}$, with $1<i \leq n$, consists of two different parts: the specification of its (absolute) position $[\vec{x}]_{1}$ with respect to $\vec{T}^{1} \backslash \vec{T}^{2}$, where $\backslash$ denotes set-theoretic difference, plus the specification of $i-1$ nested displacements $[\vec{x}]_{2}, \ldots,[\vec{x}]_{i}$ with respect to $\vec{T}^{2} \backslash \vec{T}^{3}, \ldots, \vec{T}^{i} \backslash \vec{T}^{i+1}$, respectively.

Example 2.1 Consider a temporal universe consisting of hours, minutes, and seconds. An hour is specified by its absolute value, e.g. hour 4011, a minute is specified by the hour it belongs to plus a displacement with respect to the first minute of such an hour, e.g., the sixteenth minute of hour 4011 is represented by the pair $(4011,15)$, a second is specified by the hour it belongs to plus a displacement with respect to the first second of the minute it belongs to, which in its turn is specified in the same way with respect to the hour, e.g., the third second of the sixteenth minute of hour 4011 is represented by the triplet $(4011,15,2)$.

We now define local orderings, congruences, successors, and upward and downward projections. For $i=1, \ldots, n$, the local ordering $\overrightarrow{\leq}_{i}$ between any pair of vectors $\vec{x}, \vec{y} \in$ $\vec{T}^{i}$ is defined in terms of ordering of their components.

Definition 2.2 (Local ordering) For each domain $\vec{T}^{i}$, a local ordering $\overrightarrow{\leq}_{i}$ (lexicographical ordering) is defined such that, for each pair of vectors $\vec{x}, \vec{y} \in \vec{T}^{i}$,

$$
\begin{aligned}
\vec{x} \vec{x}_{i} \vec{y} & \text { iff } \forall j\left(1 \leq j \leq i \rightarrow[\vec{x}]_{j}=[\vec{y}]_{j}\right) \vee \\
\qquad & \vee \exists j\left(1 \leq j \leq i \wedge \forall k\left(1 \leq k<j \rightarrow[\vec{x}]_{k}=[\vec{y}]_{k}\right) \wedge[\vec{x}]_{j}<[\vec{y}]_{j}\right) .
\end{aligned}
$$

A notion of local equality $\overrightarrow{=}_{i}$ of two instants $\vec{x}, \vec{y} \in \vec{T}^{i}$ can be derived immediately.

The relations of upward projection $\uparrow \subseteq \overrightarrow{\mathcal{T}} \times \overrightarrow{\mathcal{T}}$ and downward projection $\downarrow \subseteq$ $\overrightarrow{\mathcal{T}} \times \overrightarrow{\mathcal{T}}$ are defined in terms of the notions of prefix and extension, respectively.

Definition 2.3 (Prefix and extension) For all $\vec{x} \in^{\prime} \vec{T}^{i}$, a (nonempty) prefix of $\vec{x}$ is a time instant $\vec{y} \in \vec{T}^{j}$, with $1 \leq j \leq i$, such that $[\vec{x}]_{k}=[\vec{y}]_{k}$, for $k=1, \ldots, j$. For all $\vec{x} \in^{\prime} \vec{T}^{i}$, an extension of $\vec{x}$ is a time instant $\vec{y} \in^{\prime} \vec{T}^{j}$, with $i \leq j \leq n$, such that $[\vec{x}]_{k}=[\vec{y}]_{k}$, for $k=1, \ldots, i$.

Definition 2.4 (Upward and downward projections) For each pair of vectors $\vec{x}, \vec{y} \in$ $\overrightarrow{\mathcal{T}}, \uparrow(\vec{x}, \vec{y})$ holds if and only if $\vec{y}$ is a prefix of $\vec{x}$, whereas $\downarrow(\vec{x}, \vec{y})$ holds if and only if $\vec{y}$ is an extension of $\vec{x}$. 
Proposition 2.5 For any temporal domain $\vec{T}^{i}$ and any pair of vectors $\vec{x}, \vec{y} \in^{\prime} \vec{T}^{i}$, if $\vec{x}$ is not equal to $\vec{y}$, then there exists no vector $\vec{z}$ such that $\downarrow(\vec{x}, \vec{z})$ and $\downarrow(\vec{y}, \vec{z})$.

Local congruence relations $\stackrel{\equiv}{i, 2}_{i, 3}, \ldots$ between pairs of vectors $\vec{x}, \vec{y}$ belonging to the same domain $\vec{T}^{i}$ are defined in terms of (standard) congruence relations between their $i$-th components $x_{i}, y_{i}$.

Definition 2.6 (Local congruence) For each domain $\vec{T}^{i}$, each pair of vectors $\vec{x}, \vec{y} \in$ $\vec{T}^{i}$, and each natural number $d$, a local congruence relation $\equiv_{i, d}$ is defined as follows:

$$
\vec{x} \rightrightarrows_{i, d} \vec{y} \text { iff }[\vec{x}]_{i} \equiv_{d}[\vec{y}]_{i} .
$$

The apparently stronger notion of local congruence $\stackrel{\equiv}{i, d}_{i}$ between $\vec{x}, \vec{y} \in \in^{\prime} \vec{T}^{i}$ that holds whenever all the components are congruent modulo- $d$, can be defined as follows:

$$
\vec{x} ٍ_{i, d}^{\prime} \vec{y} \text { iff } \forall j\left(1 \leq j \leq i \rightarrow \vec{x} \rightrightarrows_{j, d} \vec{y}\right),
$$

where $\forall j\left(1 \leq j \leq i \rightarrow \vec{x} \rightrightarrows_{j, d} \vec{y}\right)$ is shorthand for $\vec{x} \rightrightarrows_{1, d} \vec{y} \wedge \cdots \wedge \vec{x} \rightrightarrows_{i, d} \vec{y}$. Finally, for each $\vec{T}^{i}$, a unary successor function $\overrightarrow{+}_{i} 1$ is defined.

Definition 2.7 (Local successor) Let $\vec{T}^{i}$ be a temporal domain, and $\vec{x}=\left\langle x_{1}, \ldots, x_{j}\right\rangle$, with $j \geq i$, be an element of $\vec{T}^{i}$. The application of $\overrightarrow{+}_{i} 1$ to $\vec{x}$ is defined as follows:

$$
\vec{x} \overrightarrow{+}_{i} 1= \begin{cases}\left\langle x_{1}, \ldots, x_{i}+1, \ldots, x_{j}\right\rangle & \text { if } i=1 \vee x_{i}+1<c f_{i-1, i}, \\ \left\langle x_{1}, \ldots, x_{i-1}, 0, \ldots, x_{j}\right\rangle \overrightarrow{+}_{i-1} 1 & \text { otherwise }\end{cases}
$$

where 0 and +1 are the constant 0 and the successor function of natural numbers, respectively. Notice that even if local successors are specified within a given domain, they can actually propagate to different domains.

In order to define an interpretation for the language $\mathcal{L}_{L M}^{2}$, it is useful to introduce an alternative (pseudo) vectorial representation according to which the $i$-th component of a vector denotes an absolute position with respect to $\vec{T}^{i} \backslash \vec{T}^{i+1}$. Such a representation can be automatically derived from the above given one. For each domain $\vec{T}^{i}$ and each vector $\vec{x} \in \vec{T}^{i}$, let us transform $\vec{x}$ into a (pseudo)vector $\vec{y}$ such that $[\vec{y}]_{1}=[\vec{x}]_{1}$, and, for each $j=2, \ldots, i,[\vec{y}]_{j}=\left(\ldots\left(\left([\vec{x}]_{1} \cdot c f_{1,2}+[\vec{x}]_{2}\right) \cdot c f_{2,3}+\right.\right.$ $\left.\left.[\vec{x}]_{3}\right) \ldots\right) \cdot c f_{j-1, j}+[\vec{x}]_{j}=[\vec{y}]_{j-1}+[\vec{x}]_{j}$.

Example 2.8 Assume the temporal universe of Example 2.1. The representation of the third second of the sixteenth minute of hour 4011 becomes $(4011,240675$, 14440502).

According to this alternative representation, for each $i=1, \ldots, n$, the set $\left\{\vec{x} \mid \vec{x} \in \vec{T}^{i}\right\}$ becomes a suitable subset of the product $\mathbb{N} \times \cdots \times \mathbb{N}(i$ times $)$. This representation is redundant, because each component $[\vec{y}]_{j}$ of a vector $\vec{y} \in^{\prime} \vec{T}^{i}$, with $1<j \leq i$, codifies complete information about all the components of lower index. It is indeed easy to prove that $[\vec{y}]_{j-1}$ is equal to the (unique) natural number such that $[\vec{y}]_{j-1} \cdot c f_{i-1, i} \leq$ $[\vec{y}]_{j}<\left([\vec{y}]_{j-1}+1\right) \cdot c f_{i-1, i}$. Moreover, once $[\vec{y}]_{j-1}$ has been determined, it can be used to determine $[\vec{y}]_{j-2}$, and so on, until the first component is reached. Thus, the finest component of a (pseudo)vector implicitly provides complete information about all the other components. Even if it is less elegant than the original representation, we 
will use this (pseudo) vectorial representation to make the definition of the semantic interpretation of $\mathcal{L}_{L M}^{2}$-formulas simpler.

Let $\varphi$ be a formula of $\mathcal{L}_{L M}^{2}$, with free predicate symbols $\mathrm{p}_{1}, \ldots, \mathrm{p}_{\mathrm{m}}$. Unary and binary relational symbols, and constants and function symbols, are mapped onto the corresponding components of the temporal structure. Thus, an interpretation $I$ for $\varphi$ is given by $m \cdot n$ sets $p_{1,1}^{I}, \ldots, p_{m, n}^{I} \subseteq \mathbb{N}$, where, for each set $p_{k, i}^{I}, k$ and $i$ indicate the indices of the predicate $\mathrm{p}_{\mathrm{k}}$ and of the set of time instants of $\vec{T}^{i}$, respectively. For $k=1, \ldots, m, p_{k, 1}^{I}, \ldots, p_{k, n-1}^{I}, p_{k, n}^{I}$ we define the interpretation $p_{k}^{I}$ of $\mathrm{p}_{\mathrm{k}}$ with respect to the sets $\vec{T}^{1} \backslash \vec{T}^{2}, \ldots, \vec{T}^{n-1} \backslash \vec{T}^{n}, \vec{T}^{n}$ by stipulating that $\mathrm{p}_{\mathrm{k}}$ holds on a vector $\vec{x}$ if and only if for some $i, \mathrm{p}_{\mathrm{k}}$ holds for the $i$-th component of $\vec{x}$ (i.e., $[\vec{x}]_{i} \in p_{k, i}^{I}$ ).

Example 2.9 Assume the temporal universe of the previous examples. Moreover, let $\vec{x}$ be the vector of Example 2.8. $\mathrm{p}_{\mathrm{k}}$ be a predicate symbol of $\mathcal{L}_{L M}^{2}$, and $I$ be an interpretation for $\mathcal{L}_{L M}^{2}$. According to the given definition, $\mathrm{p}_{\mathrm{k}}$ holds at $\vec{x}$ if, for instance, $240675 \in p_{k, 2}^{I}$.

2.1 Supporting basic MLTL functionalities Now that we have defined our language for talking about layered and metric temporal structures, we show how it can express the three key features of metric and layered temporal logics defined in Section 1: contextualization, and granular and metric displacement, thus showing the expressiveness of the language $\mathcal{L}_{L M}^{2}$, and its usefulness as a framework for studying metric and layered temporal logics. We will also introduce the notions of (global) temporal ordering and congruence.

Contextualization restricts the range of possible values of a given vector variable $\overrightarrow{\mathrm{x}}$ by constraining (the value of) $\overrightarrow{\mathrm{x}}$ to belong to a given domain $\vec{T}^{i}$. In $\mathcal{L}_{L M}^{2}$, contextualization is expressed by means of the $n$ unary predicates $\vec{T}^{1}(\vec{x}), \ldots, \mathrm{T}^{\mathrm{n}}(\overrightarrow{\mathrm{x}})$. Contextualization is formally defined as follows. For the sake of readability, we will use a set notation $\overrightarrow{\mathrm{x}} \in \overrightarrow{\mathrm{T}}^{\mathrm{i}}\left(\right.$ resp. $\left.\overrightarrow{\mathrm{x}} \notin \overrightarrow{\mathrm{T}}^{\mathrm{i}}\right)$ instead of $\overrightarrow{\mathrm{T}}^{\mathrm{i}}(\overrightarrow{\mathrm{x}})\left(\operatorname{resp} . \neg \overrightarrow{\mathrm{T}}^{\mathrm{i}}(\overrightarrow{\mathrm{x}})\right)$.

Definition 2.10 (Contextualization) For each vector variable $\vec{x}$ and each unary predicate $\overrightarrow{\mathrm{T}}^{\mathrm{i}}$, with $1 \leq i \leq n, \overrightarrow{\mathrm{x}} \in \overrightarrow{\mathrm{T}}^{\mathrm{i}}$ holds if and only if (the value of) $\overrightarrow{\mathrm{x}}$ belongs to the domain $\vec{T}^{i}$.

The total ordering of temporal domains defined by granularity allows us to easily prove that the formula:

$$
\forall \overrightarrow{\mathrm{x}}\left(\overrightarrow{\mathrm{x}} \in \overrightarrow{\mathrm{T}}^{\mathrm{i}} \rightarrow \forall j\left(1 \leq j \leq i \rightarrow \overrightarrow{\mathrm{x}} \in \overrightarrow{\mathrm{T}}^{j}\right)\right)
$$

is valid in all finitely-layered metric temporal structures (as usual, the consequent of the outermost implication stands for " $\vec{x} \in \vec{T}^{1} \wedge \cdots \wedge \vec{x} \in \vec{T}^{i}$ "). Contextualization also allows us to prove the following proposition.

Proposition 2.11 For each pair of vector variables $\vec{x}, \vec{y}, \vec{x}=\vec{y} \leftrightarrow \exists i\left(\vec{x} \in^{\prime} \vec{T}^{i} \wedge \vec{y} \in^{\prime}\right.$ $\left.\left.\overrightarrow{\mathrm{T}}^{i} \wedge \overrightarrow{\mathrm{x}} \overrightarrow{\mathrm{i}}_{\mathrm{i}} \overrightarrow{\mathrm{y}}\right)\right)$, where the right-hand side formula stands for " $\left(\overrightarrow{\mathrm{x}} \in^{\prime} \overrightarrow{\mathrm{T}}^{1} \wedge \overrightarrow{\mathrm{y}} \in^{\prime} \overrightarrow{\mathrm{T}}^{1} \wedge \overrightarrow{\mathrm{x}}=1\right.$ $\overrightarrow{\mathrm{y}}) \vee \cdots \vee\left(\overrightarrow{\mathrm{x}} \in \in^{\prime} \overrightarrow{\mathrm{T}}^{\mathrm{n}} \wedge \overrightarrow{\mathrm{y}} \in^{\prime} \overrightarrow{\mathrm{T}}^{\mathrm{n}} \wedge \overrightarrow{\mathrm{x}}={ }_{\mathrm{n}} \overrightarrow{\mathrm{y}}\right)$ ” and $“ \overrightarrow{\mathrm{x}} \in^{\prime} \overrightarrow{\mathrm{T}}^{\mathrm{i}} \leftrightarrow\left(\overrightarrow{\mathrm{x}} \in \overrightarrow{\mathrm{T}}^{\mathrm{i}} \wedge \overrightarrow{\mathrm{x}} \notin \mathrm{T}^{\mathrm{i}+1}\right)$."

It follows that two vectors finely belonging to different domains are distinct.

Contextualization can occur in different types of formulas. As an example, it is involved in formulas stating that there exists a time instant belonging to a given 
domain $\vec{T}^{i}$ at which a formula $\varphi$ is true, and in formulas stating that $\varphi$ is true at each instant of a given domain $\vec{T}^{i}$ (restricted quantification). These formulas take the forms $\exists \overrightarrow{\mathrm{x}}\left(\overrightarrow{\mathrm{x}} \in^{\prime} \overrightarrow{\mathrm{T}}^{i} \wedge \varphi(\overrightarrow{\mathrm{x}})\right)$ and $\forall \overrightarrow{\mathrm{x}}\left(\overrightarrow{\mathrm{x}} \in \in^{\prime} \overrightarrow{\mathrm{T}}^{\mathrm{i}} \rightarrow \varphi(\overrightarrow{\mathrm{x}})\right)$, respectively.

Definition 2.12 (Granular ordering and equivalence) We define a partial ordering $\gg$ over $\mathcal{T}$ based on the "grain-size" of vectors $(\vec{x} \gg \vec{y}$ if and only if $\vec{x}$ is coarser than $\vec{y})$ as follows:

$$
\overrightarrow{\mathrm{x}} \gg \overrightarrow{\mathrm{y}} \text { iff } \exists i, j\left(\overrightarrow{\mathrm{x}} \in \in^{\prime} \overrightarrow{\mathrm{T}}^{i} \wedge \overrightarrow{\mathrm{y}} \in^{\prime} \overrightarrow{\mathrm{T}}^{j} \wedge i<j\right),
$$

where the right-hand side formula stands for " $\left(\vec{x} \in \in^{\prime} \vec{T}^{1} \wedge \vec{y} \in \in^{\prime} \vec{T}^{2}\right) \vee\left(\vec{x} \in^{\prime} \vec{T}^{1} \wedge \vec{y} \in^{\prime}\right.$ $\left.\overrightarrow{\mathrm{T}}^{3}\right) \vee \cdots \vee\left(\overrightarrow{\mathrm{x}} \in \in^{\prime} \overrightarrow{\mathrm{T}}^{\mathrm{n}-1} \wedge \overrightarrow{\mathrm{y}} \in^{\prime} \overrightarrow{\mathrm{T}}^{\mathrm{n}}\right)$." Moreover, an equivalence relation $\sim$ over $\mathcal{T}$, such that $\vec{x} \sim \vec{y}$ if and only if $\vec{x}$ is as coarse as $\vec{y}$, can be defined as follows:

$$
\vec{x} \sim \vec{y} \text { iff } \exists i\left(\vec{x} \in^{\prime} \overrightarrow{\mathrm{T}}^{i} \wedge \overrightarrow{\mathrm{y}} \in^{\prime} \overrightarrow{\mathrm{T}}^{\mathrm{i}}\right),
$$

where the right-hand side formula stands for " $\left(\vec{x} \in^{\prime} \vec{T}^{1} \wedge \vec{y} \in^{\prime} \vec{T}^{1}\right) \vee \cdots \vee\left(\vec{x} \in^{\prime} \vec{T}^{n} \wedge\right.$ $\left.\overrightarrow{\mathrm{y}} \in^{\prime} \overrightarrow{\mathrm{T}}^{\mathrm{n}}\right) . "$

Granular displacement is directly supported by upward and downward projections. As in the case of contextualizations, we adopt a set notation $\vec{y} \in \uparrow(\vec{x})(\operatorname{resp} \cdot \vec{y} \notin \uparrow(\vec{x}))$ instead of $\uparrow(\vec{x}, \vec{y})$ (resp. $\neg \uparrow(\vec{x}, \vec{y}))$.

Definition 2.13 (Granular displacement) For each pair of vector variables $\vec{x}, \vec{y}, \vec{y} \in$ $\uparrow(\overrightarrow{\mathrm{x}})$ holds if and only if (the value of) $\overrightarrow{\mathrm{y}}$ belongs to the upward projection of (the value of) $\vec{x}$, whereas $\vec{y} \in \downarrow(\vec{x})$ holds if and only if (the value of) $\vec{y}$ belongs to the downward projection of (the value of) $\overrightarrow{\mathrm{x}}$.

Granular displacements allow one to express conditions on the belonging of an instant to the projection of another one. For instance, the constraint that $\vec{y}$ must belong to the downward projection of $\vec{x}$ is expressed by the atomic formula $\vec{y} \in \downarrow(\vec{x})$. Moreover, existential and universal quantifications under projection can be used to state that there exists $\vec{y}$ belonging to the downward projection of $\vec{x}$ such that a formula $\varphi$ is true at $\vec{y}$, as well as to state that $\varphi$ is true at each $\vec{y}$ belonging to the downward projection of $\vec{x}$ (restricted quantification). These formulas take the forms $\exists \vec{y}(\vec{y} \in \downarrow(\vec{x}) \wedge \varphi(\vec{y}))$ and $\forall \overrightarrow{\mathrm{y}}(\overrightarrow{\mathrm{y}} \in \downarrow(\overrightarrow{\mathrm{x}}) \rightarrow \varphi(\overrightarrow{\mathrm{y}}))$, respectively.

The relations $\uparrow$ and $\downarrow$ can be specialized to restrict upward and downward projections to a specific domain. For each domain $\vec{T}^{j}$, the restriction of upward (resp. downward) projection to $\vec{T}^{j}$ denoted by $\uparrow^{j} \subseteq \overrightarrow{\mathcal{T}} \times \vec{T}^{j}$ (resp. $\downarrow_{j} \subseteq \overrightarrow{\mathcal{T}} \times \vec{T}^{j}$ ) is defined as follows (according to the set notation):

$$
\overrightarrow{\mathrm{y}} \in \uparrow^{j}(\overrightarrow{\mathrm{x}}) \text { iff } \overrightarrow{\mathrm{y}} \in \uparrow(\overrightarrow{\mathrm{x}}) \wedge \overrightarrow{\mathrm{y}} \in^{\prime} \overrightarrow{\mathrm{T}}^{j}\left(\operatorname{resp} . \overrightarrow{\mathrm{y}} \in \downarrow_{j}(\overrightarrow{\mathrm{x}}) \text { iff } \overrightarrow{\mathrm{y}} \in \downarrow(\overrightarrow{\mathrm{x}}) \wedge \overrightarrow{\mathrm{y}} \in^{\prime} \overrightarrow{\mathrm{T}}^{j}\right)
$$

Proposition 2.14 For each $\vec{x} \in \overrightarrow{\mathcal{T}}$ and $1 \leq j \leq n$, there exists at most one vector $\vec{y} \in^{\prime} \vec{T}^{j}$ such that $\vec{y} \in \uparrow^{j}(\vec{x})$. More precisely, if $\vec{x} \in^{\prime} \vec{T}^{i}$ and $j \leq i$, then there exists one and only one vector $\vec{y} \in^{\prime} \vec{T}^{j}$ such that $\vec{y} \in \uparrow^{j}(\vec{x})$, whereas, if $\vec{x} \in \vec{T}^{i}$ and $i<j$, then there are no vectors $\vec{y} \in^{\prime} \vec{T}^{j}$ such that $\vec{y} \in \uparrow^{j}(\vec{x})$. Moreover, for each $\vec{x} \in \overrightarrow{\mathcal{T}}$ and $1 \leq j \leq n$, if $\vec{x} \in \in^{\prime} \vec{T}^{i}$ and $i \leq j$, then there exist $c f_{i, j}$ vectors $\vec{y} \in^{\prime} \vec{T}^{j}$ such that $\vec{y} \in \downarrow_{j}(\vec{x})$, where $c f_{i, i}=1$ and, for $j>i, c f_{i, j}=c f_{i, i+1} \cdot \ldots \cdot c f_{j-1, j}$, whereas if $\vec{x} \in^{\prime} \vec{T}^{i}$ and $j<i$, then there exist no vectors $\vec{y} \in^{\prime} \vec{T}^{j}$ such that $\vec{y} \in \downarrow_{j}(\vec{x})$. 
The following example gives a natural explanation of the proposed structure for time granularity in terms of specialized upward and downward projections.

Example 2.15 Consider a temporal universe consisting of hours, minutes, and seconds, and let $\vec{x}$ be the second represented by the triplet $(4011,15,2)$. The prefix of $\vec{x}$ with respect to $\vec{T}^{1}, \uparrow^{1}(\vec{x})$, is 4011 , its prefix with respect to $\vec{T}^{2}, \uparrow^{2}(\vec{x})$, is $(4011,15)$, the minimal extension of $\uparrow^{1}(\vec{x})$ on $\vec{T}^{2}, \downarrow_{2}\left(\uparrow^{1}(\vec{x})\right)$, is $(4011,0)$, the minimal extension of $\uparrow^{2}(\vec{x})$ on $\vec{T}^{3}, \downarrow_{3}\left(\uparrow^{2}(\vec{x})\right)$, is $(4011,15,0)$. It is immediate to see that 15 and 2 can actually be interpreted as nested displacements.

Specializations of upward and downward projections also allow us to define a relation of temporal ordering $\overrightarrow{\leq}$ over the temporal universe $\overrightarrow{\mathcal{T}}$ based on the local orderings $\overrightarrow{\leq}_{1}, \ldots, \overrightarrow{\leq}_{n}$ over $\vec{T}^{1}, \ldots, \vec{T}^{n}$.

Definition 2.16 (Temporal ordering and equivalence) A temporal ordering over $\overrightarrow{\mathcal{T}}$ is binary relation $\overrightarrow{\leq}$ such that, for each pair of vector variables $\vec{x}, \vec{y}$,

$$
\vec{x} \vec{x} \vec{y} \text { iff } \exists i, j\left(\vec{x} \in \vec{T}^{i} \wedge \vec{y} \in^{\prime} \vec{T}^{j} \wedge\left(\vec{x} \overrightarrow{\leq}_{i} \vec{y} \vee \vec{x} \overrightarrow{\leq}_{j} \vec{y}\right)\right)
$$

where the right-hand side formula is the usual shorthand. On the basis of $\overrightarrow{\leq}$, it is immediate to define a binary relation $\vec{\simeq}$ of temporal equivalence such that, for each pair of vector variables $\vec{x}, \vec{y}, \vec{x} \simeq \vec{y}$ holds if and only if $\vec{x} \vec{x} \vec{y} \wedge \vec{y} \vec{x}$ holds.

Remark 2.17 The relation of temporal equivalence induces as many classes of equivalent vectors (clusters) as the vectors belonging to the coarsest domain are. In particular, it puts in the same class a vector and all its extensions. As a consequence, it may happen that vectors that are locally ordered become members of the same class, that is, vectors that are temporally distinguishable with respect to the temporal domain they finely belong to become temporally indistinguishable with respect to coarser domains.

Just like local ordering relations, local congruence relations can be lifted to the temporal universe $\overrightarrow{\mathcal{T}}$.

Definition 2.18 (Temporal congruence) For each natural number $d$, a temporal congruence over $\overrightarrow{\mathcal{T}}$ is binary relation $\equiv_{d}$ such that, for each pair of vector variables $\overrightarrow{\mathrm{x}}, \overrightarrow{\mathrm{y}}$

$$
\overrightarrow{\mathrm{x}} \vec{\equiv}_{\mathrm{d}} \overrightarrow{\mathrm{y}} \text { iff } \exists i, j\left(\overrightarrow{\mathrm{x}} \in \in^{\prime} \overrightarrow{\mathrm{T}}^{\mathrm{i}} \wedge \overrightarrow{\mathrm{y}} \in^{\prime} \overrightarrow{\mathrm{T}}^{j} \wedge\left(\overrightarrow{\mathrm{x}} \vec{\equiv}_{i, d} \overrightarrow{\mathrm{Y}} \vee \overrightarrow{\mathrm{x}} \vec{\equiv}_{j, d} \overrightarrow{\mathrm{y}}\right)\right) .
$$

Finally, let us consider the operation of metric displacement. It can be defined in terms of the $n$ local functions $\overrightarrow{+}_{1} 1, \ldots, \overrightarrow{+}_{n} 1$ as follows.

Definition 2.19 (Metric displacement) A temporal successor function $\overrightarrow{+} 1$ is defined over $\overrightarrow{\mathcal{T}}$ such that, for each $\overrightarrow{\mathrm{x}}$, whose value belongs to $\overrightarrow{\mathcal{T}}$,

$$
\vec{y}=\vec{x} \overrightarrow{+} 1 \text { iff } \exists i\left(\vec{x} \in \vec{T}^{i} \wedge \vec{y} \overrightarrow{=}_{i} \vec{x} \overrightarrow{+}_{i} 1\right)
$$

where the formula on the right-hand side is the usual shorthand. 
It is clear that, as long as we are interested in supporting the basic functionalities of MLTL, a proper fragment of $\mathcal{L}_{L M}^{2}$ is sufficient, including (uninterpreted) unary predicate symbols, the constant symbol $\overrightarrow{0}$, the unary function symbol $\overrightarrow{+} 1$ (metric displacement), the unary predicate symbols $\overrightarrow{\mathrm{T}}^{1}, \ldots, \overrightarrow{\mathrm{T}}^{\mathrm{n}}$ (contextualizations), the binary relational symbols $\overrightarrow{\leq}$ (temporal ordering), $\uparrow$ and $\downarrow$ (granular displacements), $\vec{\equiv}_{2}, \vec{\equiv}_{3}, \ldots$ (temporal congruences), and quantification over individual variables and (uninterpreted) unary predicate symbols.

In conclusion, we point out that the theory of finitely-layered metric temporal structures does not impose any constraint on the relationships among the truth values of free predicate symbols with respect to the different domains. As an example, it may happen that a given predicate $\mathrm{p}$ is true with respect to some (all) instants of $\vec{T}^{i} \backslash T^{i+1}$ and false with respect to all instants of $T^{j} \backslash T^{j+1}$, with $1 \leq i<n, 1<j<n$, and $i<j$. This situation is described by the following example.

Example 2.20 Consider a temporal universe consisting of three temporal domains $\vec{T}^{1}, \vec{T}^{2}$, and $\vec{T}^{3}$. Assume $c f_{1,2}=6$ and $c f_{2,3}=3$. The proposition: "at the current instant an even number of atomic $\left(\vec{T}^{3}\right)$ instants have passed" always holds in $\vec{T}^{1} \backslash \vec{T}^{2}$, whereas it is true at every odd instant in $\vec{T}^{2} \backslash \vec{T}^{3}$. A proposition that it is always true in $\vec{T}^{1} \backslash \vec{T}^{2}$ and always false in $\vec{T}^{2} \backslash \vec{T}^{3}$ can now easily be built: "at the current and next instant an even number of atomic instants have passed."

However, projection relations can be used to codify specific consistency rules that, given the truth value of a formula with respect to a certain domain, allow us to constrain its truth value with respect to other domains. For lack of space we won't give the actual encoding here.

3 Decidability of finitely-layered metric temporal structures To prove the decidability of the theory of finitely-layered metric temporal structures $T_{L M}$, we will show how to define a computable function $\tau$ which translates each sentence $\varphi$ of the language $\mathcal{L}_{L M}^{2}$ for $T_{L M}$ into a sentence $\tau(\varphi)$ of $\mathcal{L}^{2}$ so that $\tau(\varphi)$ is valid (satisfiable) in S1S if and only if $\varphi$ is valid (satisfiable) in $T_{L M}$. The translation is actually performed in two steps: we first embed finitely-layered metric temporal structures into (flat) metric temporal structures; then, we reduce metric temporal structures to S1S structures.

The language $\mathcal{L}_{M}^{2}$ for the theory of (flat) metric temporal structures $T_{M}$ is the second-order language with uninterpreted unary predicate symbols, the constant symbol 0 , the unary function symbol +1 , the binary relational symbols $\leq$ and $\equiv_{2}, \equiv_{3}, \ldots$, and quantification over individual variables and unary predicate symbols. As before, $\mathcal{L}_{M}$ denotes the first-order fragment of $\mathcal{L}_{M}^{2}$. We interpret $\mathcal{L}_{M}^{2}$ over the natural numbers $\mathbb{N}$, with $\leq$ being interpreted as the usual linear order, and only consider formulas without free individual variables. Let $\varphi$ be a formula of $\mathcal{L}_{M}^{2}$ with free predicate symbols $\mathrm{p}_{1}, \ldots, \mathrm{p}_{\mathrm{m}}$. As in the case of $\mathcal{L}^{2}$, an interpretation $I$ for $\varphi$ is given by $n$ sets $p_{1}^{I}$, $\ldots, p_{m}^{I} \subseteq \mathbb{N}$. In such a case, $\mathbb{N}$ plays the role of the discrete temporal domain over which the predicates $\mathrm{p}_{1}, \ldots, \mathrm{p}_{\mathrm{n}}$ take value.

In Section 3.1. we briefly summarize existing decidability results for real-time logics. In Section 3.2 we translate each sentence $\varphi$ of $\mathcal{L}_{L M}^{2}$ into a sentence $\tau_{1}(\varphi)$ of $\mathcal{L}_{M}^{2}$; then, in Section 3.3 we translate each sentence $\psi$ of $\mathcal{L}_{M}^{2}$ into a sentence $\tau_{2}(\psi)$ of $\mathcal{L}^{2}$. The function $\tau$ is obtained composing $\tau_{1}$ and $\tau_{2}$. 
3.1 Decidability results for real-time logics Real-time logics extend linear propositional temporal logic (PTL) with an explicit notion of time. PTL is provided with a notion of state (of computation), and it is interpreted over infinite sequences of (computation) states. It is widely used to specify and verify reactive and concurrent programs/systems, e.g. Manna and Pnueli [12]. Qualitative timing constraints expressing safety and liveness properties of programs/systems can indeed be easily coded in PTL. As an example, a response property of the form "each $p$-state is followed by a $q$-state" is specified in PTL by the formula $\square(p \rightarrow \diamond q)$.

Let $\mathcal{L}^{2}$ be the second-order language with uninterpreted unary predicate symbols, the binary relational symbol $\leq$, and quantification over individual variables and unary predicate symbols, and $\mathcal{L}$ denote the first-order fragment of $\mathcal{L}^{2}$. The response property can be expressed in $\mathcal{L}$ by the formula " $\forall i(p(i) \rightarrow \exists j(i \leq j \wedge q(j)))$." PTL corresponds to a proper subset of $\mathcal{L}$, but it has the same expressive power of $\mathcal{L}$ (see Gabbay et al. [10]). $\mathcal{L}^{2}$ can be interpreted over the natural numbers $\mathbb{N}$, with $\leq$ interpreted as the usual linear order. Let $\varphi$ be a formula of $\mathcal{L}^{2}$ with free predicate symbols $p_{1}, \ldots, p_{m}$, and without free individual variables. An interpretation $I$ for $\varphi$ is given by $m$ sets $p_{1}^{I}, \ldots, p_{m}^{I} \subseteq \mathbb{N}$. Alternatively, $I$ can be described as an infinite sequence of states $\sigma=\sigma_{0}, \sigma_{1}, \ldots$, with $\sigma_{i} \subseteq\left\{p_{1}, \ldots, p_{m}\right\}$ for $i \geq 0$, such that $p_{j} \in \sigma_{i}$ if and only if $i \in p_{j}^{I}$. The set of models of $\varphi$, i.e., the set of interpretations that satisfy $\varphi$, is denoted by $\mathcal{M}(\varphi)$. PTL-formulas can be translated into $\mathcal{L}$-formulas without changing their set of models. $\mathcal{L}^{2}$ is essentially the language underlying the second-order theory of one successor S1S, because $\leq$ is definable in terms of the successor and hence inessential. Büchi connected S1S with finite automata over infinite words [2], and used this relationship to prove the decidability of S1S [3].

PTL cannot be used to specify real-time systems, because it cannot express quantitative timing constraints, such as deadlines and timing delays. To overcome this shortcoming PTL has been extended with explicit time references (Timed PTL [1]). The resulting real-time logics have explicit notions of state and time, and are interpreted over infinite sequences of timed states.

Real-time logics are characterized by three main 'parameters': the temporal domain, the primitive operations defined over it, and the time function that maps each state into its time. Different choices of the parameter values make the validity/satisfiability problems for real-time logics decidable or undecidable. Most realtime logics proposed in the literature cannot be decided, thus failing in establishing the proper balancing between expressiveness and decidability. Some of them recover decidability sacrificing completeness. In 11], Alur and Henzinger showed that the choice of taking $\mathbb{N}$ with linear order and congruence relations as the time theory and constraining the time function to be (at least weakly) monotonic makes realtime logics decidable. Formally, let $\mathcal{L}_{T}^{2}$ be the temporal extension of $\mathcal{L}^{2}$ (and $\mathcal{L}_{T}$ be its first-order fragment). Besides the state sort, $\mathcal{L}_{T}^{2}$ has a time sort, over which the constant 0 , the successor function +1 , the order relation $\leq$, and the congruence relations $\equiv_{2}, \equiv_{3}, \ldots$ are defined. Moreover, a mapping $f$ from states to times is given. Each interpretation $I$ for $\varphi \in \mathcal{L}_{T}^{2}$ can be viewed as a pair $(\sigma, \rho)$ (sequence of timed states), where $\sigma$ is an infinite sequence of states and $\rho=f^{I}$. The set of models of $\varphi$ is denoted by $\mathcal{M}_{T}(\varphi) . \mathcal{L}_{T}^{2}$-formulas can be used to express properties of sequences of timed states. As an example, a bounded response time property of the form "each $p$ - 
state is followed by a $q$-state within 1 time unit" can be expressed by the $\mathcal{L}_{T}$-formula " $\forall i(p(i) \rightarrow \exists j(i \leq j \wedge q(j) \wedge f(j) \leq f(i)+1))$ ", where $f(j) \leq f(i)+1$ holds if and only if either $f(j)=f(i)$ or $f(j)=f(i)+1$.

A formula $\varphi \in \mathcal{L}_{T}^{2}$ is satisfiable (valid) if and only if $\varphi$ is satisfied by at least one (all) sequence of timed states. The second-order theory of timed state sequences is the set of all valid $\mathcal{L}_{T}^{2}$-formulas. Timed PTL is an elementary, yet expressively complete, fragment of such a theory. Alur and Henzinger proved that this theory is decidable, by showing the finite-state character of temporal information needed to determine the truth value of a $\mathcal{L}_{T}^{2}$-formula $\varphi$ with respect to a given interpretation $I$ (information contained in $f^{I}$ ) 11 .

As an example, consider the formula expressing the bounded response time property. A sequence of timed states for this formula specifies the truth values of $p$ and $q$, and the value of $f$, at each state $i \geq 0$. For each state $i$, let us denote the time difference $f^{I}(i)-f^{I}(i-1)$, with $f^{I}(-1)=0$, by $d f^{I}(i)$. Even if $d f^{I}$ takes value over $\mathbb{N}$, to determine the truth value of the considered formula with respect to the given interpretation $I$, it suffices to know, for each state $i$, if $d f^{I}(i)$ is equal to 0 , or it is equal to 1 , or it is greater than or equal to 2 . This allows us to model $d f^{I}$ by means of three monadic predicates over the state sort Tdiff 0 , Tdiff 1 , and Tdiff $\geq 2$ only (timedifference predicates). A notion of extended state sequence for the given formula can thus be defined as a state sequence in the propositions $p, q$, Tdiff 0 , Tdiff ${ }_{1}$, and Tdiff $_{\geq 2}$ such that (i) it agrees with the original timed state sequence on $p$ and $q$, and (ii) codifies constraints on the time distances between states in terms of timedifference predicates. The same technique can be used to model time-congruence relations in terms of a finite number of monadic time-congruence predicates Tcong $_{i, j}$ over the state sort. As a general rule, it is possible to prove that, given a formula $\varphi \in \mathcal{L}_{T}^{2}$ and two interpretations $I$ and $\mathcal{I}$ for $\varphi$ with the same underlying extended state sequence, $I \in \mathcal{M}_{T}(\varphi)$ if and only if $\mathcal{I} \in \mathcal{M}_{T}(\varphi)^{2}$. This means that the extended state sequence underlying a given interpretation $I$ contains enough information to decide whether or not $\varphi$ is true with respect to $I$. Therefore, each formula $\varphi$ can be characterized in terms of the set $\mathcal{M}_{T}^{*}(\varphi)$ of the extended state sequences underlying its interpretations rather than in terms of the set $\mathcal{M}_{T}(\varphi)$.

The main outcome of Alur and Henzinger's decidability results is the method they outline. They have proved that metric temporal information (differences and congruences over the time sort) can be modeled by means of a finite set of monadic predicates over the state sort. Their proof relies on the finite-state character of (metric) temporal information, which can be expressed as follows: each temporal property that partitions an infinite set of states (instants) into a finite set of classes can be finitely modeled and it is then decidable. In the following, we generalize Alur and Henzinger's decidability results to finitely-layered metric temporal structures proving that temporal contextualization and projection can be finitely modeled.

3.2 Flattening the finitely-layered structure Let us define the translation function $\tau_{1}$ that maps each formula $\varphi \in \mathcal{L}_{L M}^{2}$ into a formula $\tau_{1}(\varphi) \in \mathcal{L}_{M}^{2}$. We preliminary replace the relations $\overrightarrow{\leq}, \vec{\equiv}_{2}, \vec{\equiv}_{3}, \ldots$ (as well as $\sim, \gg, \simeq, \uparrow^{i}, \downarrow_{i}$ ) and the function $\overrightarrow{+} 1$ by their definitions in terms of $\overrightarrow{\mathrm{T}}^{1}, \ldots, \overrightarrow{\mathrm{T}}^{\mathrm{n}}, \overrightarrow{\leq}_{1}, \ldots, \overrightarrow{\leq}_{\mathrm{n}}, \uparrow, \downarrow, \vec{\equiv}_{1, \mathrm{~d}_{1}}, \ldots, \vec{\equiv}_{\mathrm{n}, \mathrm{d}_{\mathrm{n}}}$, and $\overrightarrow{+}_{1} 1, \ldots, \overrightarrow{+}_{n} 1$. Moreover, we assume without loss of generality that terms appearing 
in atomic formulas which are not equalities are variables (notice that this is correct in view of Proposition 2.11. We first define the behavior of $\tau_{1}$ on terms; then, we specify its application to atomic formulas; finally, we show how to deal with quantifiers and logical connectives.

Terms of $\mathcal{L}_{L M}^{2}$ are defined as follows: (i) the zero vector $\overrightarrow{0}$ is a term; (ii) each vector variable $\vec{x}$ is a term; (iii) if $\vec{t}$ is a term, then $\vec{t} \overrightarrow{+}_{i} 1$ is a term; (iv) nothing else is a term. The translation of terms is performed by means of the following rules:

$$
\begin{aligned}
\tau_{1}(\overrightarrow{0}) & =0 ; \\
\tau_{1}(\vec{x}) & =x ; \\
\tau_{1}\left(\vec{t} \overrightarrow{+}_{i} m\right) & =\tau_{1}(\vec{t})+m \cdot c f_{i, n},
\end{aligned}
$$

where $\overrightarrow{+}_{i}$ m denotes $m$ superpositions of $\overrightarrow{+}_{i} 1$.

Once the preliminary replacements have been performed, atomic formulas of $\mathcal{L}_{L M}^{2}$ can only take one of the following forms:

1. $\vec{t}_{1}=\overrightarrow{\mathrm{t}}_{2}$ (term equality);

2. $\overrightarrow{\mathrm{x}} \in \overrightarrow{\mathrm{T}}^{\mathrm{i}}\left(\vec{x}\right.$ belongs to $\left.\vec{T}^{i}\right)$;

3. $\overrightarrow{\mathrm{y}} \in \uparrow(\overrightarrow{\mathrm{x}})(\vec{y}$ belongs to the upward projection of $\vec{x})$;

4. $\overrightarrow{\mathrm{y}} \in \downarrow(\overrightarrow{\mathrm{x}})$ ( $\vec{y}$ belongs to the downward projection of $\vec{x})$;

5. $\overrightarrow{\mathrm{x}} \overrightarrow{\mathrm{x}}_{\mathrm{i}} \overrightarrow{\mathrm{y}}\left(\vec{x} \in \vec{T}^{i}\right.$ does not follow $\left.\vec{y} \in \vec{T}^{i}\right)$;

6. $\overrightarrow{\mathrm{x}} \equiv_{i, \mathrm{~d}} \overrightarrow{\mathrm{y}}\left(\vec{x} \in \vec{T}^{i}\right.$ is congruent modulo $b$ with $\left.\vec{y} \in \vec{T}^{i}\right)$;

7. $\mathrm{p}_{\mathrm{k}}(\overrightarrow{\mathrm{x}})\left(p_{k}\right.$, with $1 \leq k \leq m$, holds in $\left.\vec{x}\right)$.

In the translation $\tau_{1}$ of a sentence $\varphi$, each vector variable $\overrightarrow{\mathrm{x}}$ occurring in $\varphi$ will be replaced by $n+1$ variables $\mathrm{x}_{1}, \ldots, \mathrm{x}_{\mathrm{n}}, \mathrm{x}$, where $\mathrm{x}_{1}, \ldots, \mathrm{x}_{\mathrm{n}}$ represent the starting points of the time intervals of $\mathbf{N}$ to which $\mathrm{x}$ may belong. Intuitively, $\mathrm{x}_{1}, \ldots, \mathrm{x}_{\mathrm{n}}$ represent the projections of the (absolute positions of the) components of $\vec{x}$. Whatever the formula $\varphi$ is, $\mathrm{x}_{1}, \ldots, \mathrm{x}_{\mathrm{n}}, \mathrm{x}$ must satisfy the following constraints:

(a) for $i=1, \ldots, n-1, \mathrm{x}_{\mathrm{i}} \equiv_{c f_{\mathrm{i}, \mathrm{n}}} 0$;

(b) for $i=1, \ldots, n-1, \mathrm{x}_{\mathrm{i}} \leq \mathrm{x}_{\mathrm{i}+1}<\mathrm{x}_{\mathrm{i}}+c f_{\mathrm{i}, \mathrm{n}}$;

(c) for $i=1, \ldots, n, \mathrm{x}_{\mathrm{i}} \leq \mathrm{x} \rightarrow \mathrm{x}<\mathrm{x}_{\mathrm{i}}+c f_{\mathrm{i}, \mathrm{n}}$;

(d) $\mathrm{x}_{1} \leq \mathrm{x}$,

where $c f_{i, n}=c f_{i, i+1} \cdot \ldots \cdot c f_{n-1, n}$ is the conversion factor between $\vec{T}^{i}$ and $\vec{T}^{n}$.

The first two conditions codify basic properties of temporal structures: (a) says that the time instants of $\vec{T}^{i}$ are encoded by intervals starting at $k \cdot c f_{i, n}$ and ending at $(k+1) \cdot c f_{i, n}$, for $k=0,1, \ldots$; (b) guarantees that the intervals starting at $x_{1}, \ldots, x_{n}$, are ordered by inclusion according to granularity. For $i \geq 1$, (c) will enforce $\vec{x} \in \vec{T}^{i}$ to be equivalent to $x_{i} \leq x$. Accordingly, (d) expresses the fact that, for every $\vec{x}, \vec{x} \in \vec{T}^{1}$.

For every vector variable $\vec{x}$, the formula $\xi\left(x_{1}, \ldots, x_{n}, x\right)$ defined as:

$$
\begin{aligned}
\bigwedge_{i=1}^{n-1}\left(x_{i}\right. & \left.\equiv{ }_{c f_{i, n}} 0 \wedge x_{i} \leq x_{i+1}<x_{i}+c f_{i, n}\right) \wedge \\
& \wedge \bigwedge_{i=1}^{n}\left(x_{i} \leq x \rightarrow x<x_{i}+c f_{i, n}\right) \wedge x_{1} \leq x,
\end{aligned}
$$


will be introduced by the translation in order to guarantee (a)-(d) to hold. Since each individual variable $\overrightarrow{\mathrm{x}}$ occurring in $\varphi$ is quantified, $\xi\left(\mathrm{x}_{1}, \ldots, \mathrm{x}_{\mathrm{n}}, \mathrm{x}\right)$ will be introduced during the translation of $\forall \vec{x}$ or of $\exists \vec{x}$ to constrain the relationships among $\mathrm{x}_{1}, \ldots, \mathrm{x}_{\mathrm{n}}, \mathrm{x}$.

3.2.1 Term equality In view of Proposition 2.11 atomic formulas of the form (1) expressing term equality are translated as follows:

$$
\tau_{1}\left(\overrightarrow{\mathrm{t}}_{1}=\overrightarrow{\mathrm{t}}_{2}\right)=\tau_{1}\left(\overrightarrow{\mathrm{t}}_{1}\right)=\tau_{1}\left(\overrightarrow{\mathrm{t}}_{2}\right)
$$

3.2.2 Contextualizations Atomic formulas of the form (2) constrain (the value of) $\overrightarrow{\mathrm{x}}$ to belong to a specific domain $\vec{T}^{i}$ of the temporal universe. The application of $\tau_{1}$, together with condition (c) in $\xi\left(\mathrm{x}_{1}, \ldots, \mathrm{x}_{\mathrm{n}}, \mathrm{x}\right)$, restricts the set of admissible values for $\mathrm{x}$ to the interval $\left[x_{i}, x_{i}+c f_{i, n}\right)$.

Accordingly, the translation of $\overrightarrow{\mathrm{x}} \in \overrightarrow{\mathrm{T}}^{\mathrm{i}}$, with $1 \leq i \leq n$, is defined as follows:

$$
\tau_{1}\left(\overrightarrow{\mathrm{x}} \in \overrightarrow{\mathrm{T}}^{\mathrm{i}}\right)=\mathrm{x}_{\mathrm{i}} \leq \mathrm{x} .
$$

It follows from (c) that $x_{j} \leq x$, for $j=1, \ldots, i-1$. Moreover, in view of the above defined translation of contextualizations and of the compositionality of $\tau_{1}$ with respect to negations, the translation of the fine membership of $\overrightarrow{\mathrm{x}}$ to $\overrightarrow{\mathrm{T}}^{\mathrm{i}}$ will result in $x_{i} \leq x$ and $x<x_{i+1}$.

3.2.3 Upward and downward projections Atomic formulas of forms (3) and (4) relate (the values of) $\vec{x}$ and $\vec{y}$ possibly belonging to different domains. Their translation is more complex, because neither the domain of $\vec{x}$ nor the domain of $\vec{y}$ are known in advance, and therefore the translation must encompass all possible cases. First, (the value of) $\overrightarrow{\mathrm{x}}$ can belong to any domain. Moreover, if (the value of) $\overrightarrow{\mathrm{x}}$ belongs to $\vec{T}^{i}$ and (the value of) $\overrightarrow{\mathrm{y}}$ belongs to its upward (resp. downward) projection, then (the value of) $\vec{y}$ can belong to any domain $\vec{T}^{j}$ coarser (resp. finer) than $\vec{T}^{i}$. The translations of $\overrightarrow{\mathrm{y}} \in \uparrow(\overrightarrow{\mathrm{x}})$ and $\overrightarrow{\mathrm{y}} \in \downarrow(\overrightarrow{\mathrm{x}})$ are therefore defined as follows:

$$
\begin{aligned}
& \tau_{1}(\overrightarrow{\mathrm{y}} \in \uparrow(\overrightarrow{\mathrm{x}}))=\exists i, j\left(\mathrm{x}_{\mathrm{i}} \leq \mathrm{x}<\mathrm{x}_{\mathrm{i}+1} \wedge \mathrm{y}_{j} \leq \mathrm{y}<\mathrm{y}_{\mathrm{j}+1} \wedge \mathrm{j} \leq \mathrm{i} \wedge \mathrm{x}_{\mathrm{j}}=\mathrm{y}_{\mathrm{j}}\right), \\
& \tau_{1}(\overrightarrow{\mathrm{y}} \in \downarrow(\overrightarrow{\mathrm{x}}))=\exists i, j\left(\mathrm{x}_{\mathrm{i}} \leq \mathrm{x}<\mathrm{x}_{\mathrm{i}+1} \wedge \mathrm{y}_{\mathrm{j}} \leq \mathrm{y}<\mathrm{y}_{\mathrm{j}+1} \wedge \mathrm{i} \leq \mathrm{j} \wedge \mathrm{x}_{\mathrm{i}}=\mathrm{y}_{\mathrm{i}}\right),
\end{aligned}
$$

where both formulas are shorthands for finite disjunctions as usual.

3.2.4 Local orderings and congruences Atomic formulas of the form (5) constrain the ordering of (the values of) $\vec{x}$ and $\vec{y}$ finely belonging to the same temporal domain $\vec{T}^{i}$. The ordering relation between $\overrightarrow{\mathrm{x}}$ and $\overrightarrow{\mathrm{y}}$ is translated into an ordering relation between the starting points of the corresponding intervals:

$$
\tau_{1}\left(\overrightarrow{\mathrm{x}} \overrightarrow{\underline{x}}_{\mathrm{i}} \overrightarrow{\mathrm{y}}\right)=\mathrm{x}_{\mathrm{i}} \leq \mathrm{y}_{\mathrm{i}}
$$

Atomic formulas of the form (6) constrain (the values of) $\vec{x}, \vec{y}$ finely belonging to the same temporal domain $\vec{T}^{i}$ to belong to the same congruence class modulo- $d$ with respect to $\vec{T}^{i}$. The translation constrains the starting points of the corresponding intervals to belong to the same congruence class modulo- $\left(d \cdot c f_{\text {in }}\right)$ with respect to $\mathbb{N}$ :

$$
\tau_{1}\left(\overrightarrow{\mathrm{x}} \equiv_{\mathrm{i}, \mathrm{d}} \overrightarrow{\mathrm{y}}\right)=\mathrm{x}_{\mathrm{i}} \equiv_{\mathrm{d} \cdot c f_{\mathrm{i}, \mathrm{n}}} \mathrm{y}_{\mathrm{i}} .
$$


Remark 3.1 We assume that $\overrightarrow{+}_{i} 1, \overrightarrow{\leq}_{i}$, and $\vec{\equiv}_{i, d}$ are applied to variables of the proper type. Notice, however, that we do not need to check the domains of the arguments of $\overrightarrow{+}_{i} 1, \overrightarrow{\leq}_{i}$, or $\vec{\equiv}_{i, d}$ whenever they are generated by the expansion of $\overrightarrow{+} 1, \overrightarrow{\leq}$, and $\vec{\equiv}_{d}$, respectively. This fact guarantees that type constraints are satisfied.

3.2.5 Predicates Atomic formulas of the form (7) state the truth of a predicate $\mathrm{p}_{\mathrm{k}}$ at $\vec{x}$. As we have already noticed, it may happen that, for example, there exist two domains $\vec{T}^{i}$ and $\vec{T}^{j}$, with $i<j$, and a predicate $\mathrm{p}_{\mathrm{k}}$ such that $\mathrm{p}_{\mathrm{k}}$ holds at a given $\vec{x} \in^{\prime} \vec{T}^{i}$ and $p_{\mathrm{k}}$ does not hold at any $\vec{y} \in^{\prime} \vec{T}^{j}$ such that $\vec{y} \in^{\prime} \downarrow_{j}(\vec{x})$. As a consequence, for each predicate symbol $\mathrm{p}_{\mathrm{k}}$ we need to introduce $n$ distinct predicate symbols $\mathrm{p}_{\mathrm{k}, 1}, \ldots \mathrm{p}_{\mathrm{k}, \mathrm{n}}$ to model the truth of $\mathrm{p}_{\mathrm{k}}$ with respect to the sets $\vec{T}^{1} \backslash \vec{T}^{2}, \ldots, \vec{T}^{n}$, respectively. ${ }^{3}$

Besides replacing the predicate symbol $\mathrm{p}_{\mathrm{k}}$ by the $n$ predicate symbols $\mathrm{p}_{\mathrm{k}, 1}, \ldots$ $p_{k, n}$, the translation states that there exists an index $i$ such that $\mathrm{x}$ is greater than or equal to $\mathrm{x}_{\mathrm{i}}$ and $\mathrm{p}_{\mathrm{k}, \mathrm{i}}$ holds at $\mathrm{x}$ :

$$
\tau_{1}\left(\mathrm{p}_{\mathrm{k}}(\overrightarrow{\mathrm{x}})\right)=\exists \mathrm{i}\left(\mathrm{x}_{\mathrm{i}} \leq \mathrm{x} \wedge \mathrm{p}_{\mathrm{k}, \mathrm{i}}(\mathrm{x})\right),
$$

where the translation is a shorthand for a finite disjunction.

3.2.6 Quantifiers and logical connectives To generalize the translation function to any $\mathcal{L}_{L M}^{2}$ sentence, we must define its behavior on quantifiers and logical connectives. Each quantification of individual variables $\forall \overrightarrow{\mathrm{x}}$ (resp. $\exists \overrightarrow{\mathrm{x}}$ ) is split into $n$ quantifications $\forall \mathrm{x}_{1}, \ldots, \forall \mathrm{x}_{\mathrm{n}}$ (resp. $\exists \mathrm{x}_{1}, \ldots, \exists \mathrm{x}_{\mathrm{n}}$ ). Moreover, a nested existential quantification of the variable $\mathrm{x}$ is added. Finally, the formula $\xi\left(\mathrm{x}_{1}, \ldots, \mathrm{x}_{\mathrm{n}}, \mathrm{x}\right)$ is inserted to restrict the set of admissible values for $\mathrm{x}_{1}, \ldots, \mathrm{x}_{\mathrm{n}}, \mathrm{x}$.

The translation of quantified formulas is thus defined as follows:

$$
\begin{aligned}
\tau_{1}(\forall \overrightarrow{\mathrm{x}} \varphi) & =\forall \mathrm{x}_{1}, \ldots, \forall \mathrm{x}_{\mathrm{n}} \exists \mathrm{x}\left(\left(\xi\left(\mathrm{x}_{1}, \ldots, \mathrm{x}_{\mathrm{n}}, \mathrm{x}\right) \rightarrow \tau_{1}(\varphi)\right),\right. \\
\tau_{1}(\exists \overrightarrow{\mathrm{x}} \varphi) & =\exists \mathrm{x}_{1}, \ldots, \exists \mathrm{x}_{\mathrm{n}} \exists \mathrm{x}\left(\xi\left(\mathrm{x}_{1}, \ldots, \mathrm{x}_{\mathrm{n}}, \mathrm{x}\right) \wedge \tau_{1}(\varphi)\right)
\end{aligned}
$$

Remark 3.2 The translation of quantifications over individual variables provides us with the set of all variables that can possibly occur in the translation of the formula in their scope. Which ones of these variables will actually come into play in the translation of the quantified formula depends on the contextualizations contained in the formula (if any).

Each quantification of predicate variables $\exists \mathrm{p}_{\mathrm{k}}$ (resp. $\forall \mathrm{p}_{\mathrm{k}}$ ) is split into $n$ quantifications $\exists \mathrm{p}_{\mathrm{k}, 1}, \ldots, \exists \mathrm{p}_{\mathrm{k}, \mathrm{n}}$ (resp. $\forall \mathrm{p}_{\mathrm{k}, 1}, \ldots, \forall \mathrm{p}_{\mathrm{k}, \mathrm{n}}$ ). The corresponding translation of quantified formulas (existential case) is defined as follows:

$$
\tau_{1}\left(\exists \mathrm{p}_{\mathrm{k}} \varphi\right)=\exists \mathrm{p}_{\mathrm{k}, 1}, \ldots, \exists \mathrm{p}_{\mathrm{k}, \mathrm{n}} \tau_{1}(\varphi) .
$$

Finally, the translation distributes over the logical connectives.

The definition we have adopted for the validity of a given predicate $\mathrm{p}_{\mathrm{k}}$ on a given vector $\vec{x}$ hides an existential quantifier ranging over the components of $\vec{x}\left(p_{k}(\vec{x})\right.$ holds if and only if for some $j, \mathrm{p}_{\mathrm{k}}$ holds on the $j$-th component of $\overrightarrow{\mathrm{x}}$ ). It is useful to compare the translations of $\mathrm{p}_{\mathrm{k}}(\overrightarrow{\mathrm{x}})$ and $\neg \mathrm{p}_{\mathrm{k}}(\overrightarrow{\mathrm{x}})$ to see how $\tau_{1}$ deals with the different strength of 
positive and negative assertions. In the first case, the resulting formula says that there exists $i$ such that $\mathrm{p}_{\mathrm{k}, \mathrm{i}}$ holds at $\mathrm{x}$ and $\mathrm{x}_{\mathrm{i}} \leq \mathrm{x}$; in the second case, the resulting formula says that, for all $i$, either $\mathrm{x}<\mathrm{x}_{\mathrm{i}}$ or $\mathrm{p}_{\mathrm{k}, \mathrm{i}}$ does not hold at $\mathrm{x}$ (or both). Therefore, the only way to say that there exists $i$ such that $\neg \mathrm{p}_{\mathrm{k}, \mathrm{i}}$ holds at $\mathrm{x}$ and $\mathrm{x}_{\mathrm{i}} \leq \mathrm{x}$ is replacing $\neg \mathrm{p}_{\mathrm{k}}(\overrightarrow{\mathrm{x}})$ by nonp $\mathrm{p}_{\mathrm{k}}(\overrightarrow{\mathrm{x}})$ in the formula $\neg \mathrm{p}_{\mathrm{k}}(\overrightarrow{\mathrm{x}})$, where nonp $\mathrm{p}_{\mathrm{k}}$ is a new predicate such that, for all $\vec{x}$, nonp $p_{k}$ holds at $\vec{x}$ if and only if $p_{k}$ does not hold at $\vec{x}$.

Let us now prove that $\tau_{1}$ preserves the satisfiability (validity) of sentences of $\mathcal{L}_{L M}^{2}$. By induction on formulas we prove a more general preservation result for generic formulas, instead of just sentences. We need a semantic counterpart of the translation function $\tau_{1}$, mapping interpretations $I$ for $\mathcal{L}_{L M}^{2}$ into interpretations $\mathcal{g}=$ $\tau_{1}(I)$ for $\mathcal{L}_{M}^{2}$.

Definition 3.3 Let $I$ be an interpretation for $\mathcal{L}_{L M}^{2}$. The interpretation $\tau_{1}(I)$ for $\mathcal{L}_{M}^{2}$ is defined as follows. For all free predicate symbols $\mathrm{p}_{\mathrm{k}}$ in $\mathcal{L}_{L M}^{2}$,

$$
p_{k, i}^{\tau_{1}(I)}=\left\{x \mid\left\lfloor x / c f_{i, n}\right\rfloor \in p_{k, i}^{I}\right\}
$$

where $p_{k, i}^{\tau_{1}(I)}$ is the interpretation of the predicate $\mathrm{p}_{\mathrm{k}, \mathrm{i}} \in \mathcal{L}_{M}^{2}$, and $p_{k, i}^{I}$ is the restriction of the interpretation of the predicate $\mathrm{p}_{\mathrm{k}} \in \mathcal{L}_{L M}^{2}$ to the domain $\vec{T}^{i} \backslash \vec{T}^{i+1}$.

In the following we prove that the only $\mathcal{L}_{M}^{2}$-interpretations we need to consider in order to check satisfiability/validity are those of the form $\tau_{1}(I)$, for some interpretation $I$ for $\mathcal{L}_{L M}^{2}$.

First of all, we show that for any sentence $\varphi \in \mathcal{L}_{L M}^{2}$ and any interpretation $I$ for $\varphi, I$ satisfies $\varphi$ if and only if $\tau_{1}(I)$ satisfies $\tau_{1}(\varphi) \in \mathcal{L}_{M}^{2}$.

Lemma 3.4 Let I be an interpretation for the formula $\varphi \in \mathcal{L}_{L M}^{2}$, with free individual variables $\vec{x}^{1}, \ldots, \vec{x}^{l}$. It holds that:

$$
I \models \varphi\left(\overrightarrow{\mathrm{x}}^{1}, \ldots, \overrightarrow{\mathrm{x}}^{1}\right) \text { iff } \tau_{1}(I) \models \bigwedge_{\mathrm{h}=1}^{1} \xi\left(\mathrm{x}_{1}^{\mathrm{h}}, \ldots, \mathrm{x}_{\mathrm{n}}^{\mathrm{h}}, \mathrm{x}^{\mathrm{h}}\right) \wedge \tau_{1}\left(\varphi\left(\overrightarrow{\mathrm{x}}^{1}, \ldots, \overrightarrow{\mathrm{x}}^{1}\right)\right) .
$$

Proof: The proof is by induction on $\varphi$. The case of atomic formulas of the form (1) is straightforward. If $\varphi$ is an atomic formula of form (2-7), it is sufficient to observe that for any satisfying assignment

$$
I(\overrightarrow{\mathrm{x}})=\overrightarrow{t^{1}}=\left(t_{1}^{1}, \ldots, t_{i}^{1}\right), I(\overrightarrow{\mathrm{y}})=\overrightarrow{t^{2}}=\left(t_{1}^{2}, \ldots, t_{j}^{2}\right),
$$

for $\varphi$ with respect to $I$, the assignment

$$
\begin{aligned}
\tau_{1}(I)\left(\mathrm{x}_{1}\right) & =t_{1}^{1} \cdot c f_{1, n}, \\
& \vdots \\
\tau_{1}(I)\left(\mathrm{x}_{\mathrm{i}}\right) & =\tau_{1}(I)(\mathrm{x})=t_{i}^{1} \cdot c f_{i, n} \\
\tau_{1}(I)\left(\mathrm{x}_{\mathrm{i}+1}\right) & =\ldots=\tau_{1}(I)\left(\mathrm{x}_{\mathrm{n}}\right)=\tau_{1}(I)\left(\mathrm{x}_{\mathrm{i}}\right)+c f_{i, n}, \\
\tau_{1}(I)\left(\mathrm{y}_{1}\right) & =t_{1}^{2} \cdot c f_{1, n} \\
& \vdots \\
\tau_{1}(I)\left(\mathrm{y}_{\mathrm{j}}\right) & =\tau_{1}(I)(\mathrm{y})=t_{j}^{2} \cdot c f_{j, n} \\
\tau_{1}(I)\left(\mathrm{y}_{\mathrm{j}+1}\right) & =\ldots=\tau_{1}(I)\left(\mathrm{y}_{\mathrm{n}}\right)=\tau_{1}(I)\left(\mathrm{y}_{\mathrm{j}}\right)+c f_{j, n},
\end{aligned}
$$


satisfies $\xi\left(\mathrm{x}_{1}, \ldots, \mathrm{x}_{\mathrm{n}}, \mathrm{x}\right) \wedge \xi\left(\mathrm{y}_{1}, \ldots, \mathrm{y}_{\mathrm{n}}, \mathrm{y}\right) \wedge \tau_{1}(\varphi)$ with respect to $\tau_{1}(I)$.

As examples, consider the cases in which $\varphi$ is an atom of the form either $\mathrm{p}_{\mathrm{k}}(\overrightarrow{\mathrm{x}})$ or $\overrightarrow{\mathrm{y}} \in \uparrow(\overrightarrow{\mathrm{x}})$. In the first case, $I \models \mathrm{p}_{\mathrm{k}}(\overrightarrow{\mathrm{x}})$ is equivalent to say that there exists a vector $\vec{t}=\left(t_{1}, \ldots, t_{i}, \ldots, t_{h}\right)$ such that $t_{i} \in p_{k, i}^{I}$; therefore, by definition of $\tau_{1}(I)$,

$$
p_{k, i}^{\tau_{1}(I)}\left(t_{i} \cdot c f_{i, n}\right)
$$

holds, and hence the formula $\xi\left(\mathrm{x}_{1}, \ldots, \mathrm{x}_{\mathrm{n}}, \mathrm{x}\right) \wedge \exists \mathrm{i}\left(\mathrm{x}_{\mathrm{i}} \leq \mathrm{x} \wedge \mathrm{p}_{\mathrm{k}, \mathrm{i}}(\mathrm{x})\right)$ is satisfied with respect to $\tau_{1}(I)$ by the above defined assignment. Let us now consider the case of $\varphi=\overrightarrow{\mathrm{y}} \in \uparrow(\overrightarrow{\mathrm{x}})$. From $I=\overrightarrow{\mathrm{y}} \in \uparrow(\overrightarrow{\mathrm{x}})$, it follows that there exist $\overrightarrow{t^{1}} \in{ }^{\prime} T^{i}$ and $\overrightarrow{t^{2}} \in \epsilon^{\prime} T^{j}$ such that $j \leq i$ and the assignment $I(\overrightarrow{\mathrm{x}})=\vec{t}^{1}, I(\overrightarrow{\mathrm{y}})=\overrightarrow{t^{2}}$ satisfies $\varphi$. In these hypotheses, $t_{j}^{2}=\left\lfloor t_{i}^{1} / c f_{j, i}\right\rfloor$. To see that the translated formula

$$
\begin{aligned}
& \xi\left(\mathrm{x}_{1}, \ldots, \mathrm{x}_{\mathrm{n}}, \mathrm{x}\right) \wedge \xi\left(\mathrm{y}_{1}, \ldots, \mathrm{y}_{\mathrm{n}}, \mathrm{y}\right) \\
& \quad \exists i, j\left(\mathrm{x}_{\mathrm{i}} \leq \mathrm{x} \wedge \mathrm{x}<\mathrm{x}_{\mathrm{i}+1} \wedge \mathrm{y}_{j} \leq \mathrm{y} \wedge \mathrm{y}<\mathrm{y}_{\mathrm{i}+1} \wedge \mathrm{j} \leq \mathrm{i} \wedge \mathrm{x}_{\mathrm{j}}=\mathrm{y}_{\mathrm{j}}\right),
\end{aligned}
$$

is satisfied by the above defined assignment, recall that $\tau_{1}(I)(\mathrm{x})=\tau_{1}(I)\left(\mathrm{x}_{\mathrm{i}}\right)=t_{i}^{1}$. $c f_{i, n}$ and $\tau_{1}(I)(\mathrm{y})=\tau_{1}(I)\left(\mathrm{y}_{j}\right)=t_{j}^{2} \cdot c f_{j, n}$. It is straightforward to prove that the formula is satisfied. In particular, notice that

$$
t_{j}^{1} \cdot c f_{j, n}=\left\lfloor t_{i}^{1} \cdot c f_{i, n} / c f_{j, n}\right\rfloor \cdot c f_{j, n}=\left\lfloor t_{i}^{1} / c f_{j, i}\right\rfloor \cdot c f_{j, n}=t_{j}^{2} \cdot c f_{j, n} .
$$

Conversely, given a satisfying assignment

$$
\begin{aligned}
\tau_{1}(I)\left(\mathrm{x}_{1}\right) & =a_{1} \\
& \vdots \\
\tau_{1}(I)\left(\mathrm{x}_{\mathrm{n}}\right) & =a_{n} \\
\tau_{1}(I)(\mathrm{x}) & =a \\
\tau_{1}(I)\left(\mathrm{y}_{1}\right) & =b_{1} \\
& \vdots \\
\tau_{1}(I)\left(\mathrm{y}_{\mathrm{n}}\right) & =b_{n} \\
\tau_{1}(I)(\mathrm{y}) & =b
\end{aligned}
$$

for $\xi\left(\mathrm{x}_{1}, \ldots, \mathrm{x}_{\mathrm{n}}, \mathrm{x}\right) \wedge \xi\left(\mathrm{y}_{1}, \ldots, \mathrm{y}_{\mathrm{n}}, \mathrm{y}\right) \wedge \tau_{1}(\varphi)$ with respect to $\tau_{1}(I)$, a satisfying assignment for $\varphi$ with respect to $I$ can be obtained as follows. Let $i$ and $j$ be such that $a_{i} \leq a<a_{i+1}$ and $b_{j} \leq b<b_{j+1}$; the vectors $\vec{t}^{1}$ and $\overrightarrow{t^{2}}$ defined as

$$
\overrightarrow{t^{1}}=\left(a_{1} / c f_{1, n}, \ldots, a_{i} / c f_{i, n}\right), \overrightarrow{t^{2}}=\left(b_{1} / c f_{1, n}, \ldots, b_{j} / c f_{j, n}\right),
$$

satisfy $\varphi$ with respect to $I$.

For existential quantification over individual variables notice that

$$
I \models \exists \overrightarrow{\mathrm{x}}^{1} \varphi\left(\overrightarrow{\mathrm{x}}^{1}, \ldots, \overrightarrow{\mathrm{x}}^{1}\right)
$$

is equivalent to $I \models \varphi\left(\overrightarrow{\mathrm{x}}^{1}, \ldots, \overrightarrow{\mathrm{x}}^{1}\right)$, and the thesis follows directly from the inductive hypothesis and the definition of $\tau_{1}$. 
For existential quantification on predicate variables we must show that

$$
\begin{aligned}
& I \models \exists \mathrm{p}_{\mathrm{k}} \psi\left(\overrightarrow{\mathrm{x}}^{1}, \ldots, \overrightarrow{\mathrm{x}}^{1}\right) \text { iff } \\
& \qquad \tau_{1}(I) \models \bigwedge_{\mathrm{h}=1}^{1} \xi\left(\mathrm{x}_{1}^{\mathrm{h}}, \ldots, \mathrm{x}_{\mathrm{n}}^{\mathrm{h}}, \mathrm{x}^{\mathrm{h}}\right) \wedge \tau_{1}\left(\exists \mathrm{p}_{\mathrm{k}} \psi\left(\overrightarrow{\mathrm{x}}^{1}, \ldots, \overrightarrow{\mathrm{x}}^{1}\right)\right) .
\end{aligned}
$$

If the left-hand side of $(*)$ holds, then $I^{p_{k}} \models \psi\left(\overrightarrow{\mathrm{x}}^{1}, \ldots, \overrightarrow{\mathrm{x}}^{1}\right)$ for some $I^{p_{k}}$ extension of $I$ to the predicate symbol $\mathrm{p}_{\mathrm{k}}$. Hence, by inductive hypothesis, we have

$$
\tau_{1}\left(I^{p_{k}}\right) \models \bigwedge_{\mathrm{h}=1}^{\mathrm{l}} \xi\left(\mathrm{x}_{1}^{\mathrm{h}}, \ldots, \mathrm{x}_{\mathrm{n}}^{\mathrm{h}}, \mathrm{x}^{\mathrm{h}}\right) \wedge \tau_{1}\left(\psi\left(\overrightarrow{\mathrm{x}}^{1}, \ldots, \overrightarrow{\mathrm{x}}^{\mathrm{l}}\right)\right),
$$

from which it is easy to see that the right-hand side of $(*)$ holds.

Conversely, if the right-hand side of $(*)$ holds, then there exists an extension of the interpretation $\tau_{1}(I)$ to the predicates $\mathrm{p}_{\mathrm{k}, 1}, \ldots, \mathrm{p}_{\mathrm{k}, \mathrm{n}}$, that we can denote by $\tau_{1}(I)^{p_{k}}$, such that $\tau_{1}(I)^{p_{k}} \models \bigwedge_{\mathrm{h}=1}^{\mathrm{l}} \xi\left(\mathrm{x}_{1}^{\mathrm{h}}, \ldots, \mathrm{x}_{\mathrm{n}}^{\mathrm{h}}, \mathrm{x}^{\mathrm{h}}\right) \wedge \tau_{1}\left(\psi\left(\overrightarrow{\mathrm{x}}^{1}, \ldots, \overrightarrow{\mathrm{x}}^{1}\right)\right)$. In this case, we can conclude that

$$
\left.\tau_{1}\left(I^{p_{k}}\right)\right) \models \bigwedge_{\mathrm{h}=1}^{1} \xi\left(\mathrm{x}_{1}^{\mathrm{h}}, \ldots, \mathrm{x}_{\mathrm{n}}^{\mathrm{h}}, \mathrm{x}^{\mathrm{h}}\right) \wedge \tau_{1}\left(\psi\left(\overrightarrow{\mathrm{x}}^{1}, \ldots, \overrightarrow{\mathrm{x}}^{1}\right)\right),
$$

where $I^{p_{k}}$ is the extension of $I$ to the predicate symbol $\mathrm{p}_{\mathrm{k}}$ (to see this, one can use Lemmas 3.6 and 3.8 below and observe that $\left.\epsilon\left(\pi\left(\tau_{1}(I)^{p_{k}}\right)\right)=\tau_{1}\left(I^{p_{k}}\right)\right)$. Now, by inductive hypothesis, $I^{p_{k}} \models \psi\left(\overrightarrow{\mathrm{x}}^{1}, \ldots, \overrightarrow{\mathrm{x}}^{1}\right)$, and hence $I \models \exists \mathrm{p}_{\mathrm{k}} \psi\left(\overrightarrow{\mathrm{x}}^{1}, \ldots, \overrightarrow{\mathrm{x}}^{1}\right)$.

If $\varphi\left(\overrightarrow{\mathrm{x}}^{1}, \ldots, \overrightarrow{\mathrm{x}}^{1}\right)$ is of the form $\neg \psi\left(\overrightarrow{\mathrm{x}}^{1}, \ldots, \overrightarrow{\mathrm{x}}^{1}\right)$, from the inductive hypothesis, it follows that $I \models \varphi$ if and only if

$$
\tau_{1}(I) \not \models \bigwedge_{\mathrm{h}=1}^{1} \xi\left(\mathrm{x}_{1}^{\mathrm{h}}, \ldots, \mathrm{x}_{\mathrm{n}}^{\mathrm{h}}, \mathrm{x}^{\mathrm{h}}\right) \wedge \tau_{1}\left(\psi\left(\mathrm{x}^{1}, \ldots, \mathrm{x}^{\mathrm{l}}\right)\right),
$$

namely, $\tau_{1}(I) \models \bigvee_{\mathrm{h}=1}^{\mathrm{l}} \neg \xi\left(\mathrm{x}_{1}^{\mathrm{h}}, \ldots, \mathrm{x}_{\mathrm{n}}^{\mathrm{h}}, \mathrm{x}^{\mathrm{h}}\right) \vee \tau_{1}\left(\neg \psi\left(\mathrm{x}^{1}, \ldots, \mathrm{x}^{1}\right)\right)$. However, as $\tau_{1}(I) \models \bigwedge_{\mathrm{h}=1}^{\mathrm{l}} \xi\left(\mathrm{x}_{1}^{\mathrm{h}}, \ldots, \mathrm{x}_{\mathrm{n}}^{\mathrm{h}}, \mathrm{x}^{\mathrm{h}}\right)$, we have that the above is equivalent to $\tau_{1}(I) \models$ $\bigwedge_{\mathrm{h}=1}^{\mathrm{l}} \xi\left(\mathrm{x}_{1}^{\mathrm{h}}, \ldots, \mathrm{x}_{\mathrm{n}}^{\mathrm{h}}, \mathrm{x}^{\mathrm{h}}\right) \wedge \tau_{1}\left(\neg \psi\left(\mathrm{x}^{1}, \ldots, \mathrm{x}^{\mathrm{l}}\right)\right)$.

Finally, the case of conjunctions of formulas follows easily from the inductive hypothesis and the fact that $\tau_{1}$ distributes over conjunctions. This concludes the proof. $^{4}$

On the ground of the previous result, we have that $\tau_{1}$ preserves satisfiability. In order to prove that also validity is preserved, we show that for any sentence $\varphi \in \mathcal{L}_{L M}^{2}$ and any interpretation $\mathcal{I}$ for $\tau_{1}(\varphi)$, there exist an interpretation $\mathcal{I}^{\prime}$ for $\tau_{1}(\varphi)$ and an interpretation $I$ for $\varphi$ such that $\mathcal{I} \models \tau_{1}(\varphi)$ iff $\mathcal{I}^{\prime} \models \tau_{1}(\varphi)$ and $\mathcal{I}^{\prime}=\tau_{1}(I)$. We will prove that, for any formula $\varphi \in \mathcal{L}_{L M}^{2}$, with free individual variables $\overrightarrow{\mathrm{x}}^{1}, \ldots, \overrightarrow{\mathrm{x}}^{1}$,

$$
\begin{aligned}
\mathcal{I} \models & \bigwedge_{\mathrm{h}=1}^{\mathrm{l}} \xi\left(\mathrm{x}_{1}^{\mathrm{h}}, \ldots, \mathrm{x}_{\mathrm{n}}^{\mathrm{h}}, \mathrm{x}^{\mathrm{h}}\right) \wedge \tau_{1}\left(\varphi\left(\overrightarrow{\mathrm{x}}^{1}, \ldots, \overrightarrow{\mathrm{x}}^{1}\right)\right) \text { iff } \\
& \mathcal{I}^{\prime} \models \bigwedge_{\mathrm{h}=1}^{1} \xi\left(\mathrm{x}_{1}^{\mathrm{h}}, \ldots, \mathrm{x}_{\mathrm{n}}^{\mathrm{h}}, \mathrm{x}^{\mathrm{h}}\right) \wedge \tau_{1}\left(\varphi\left(\overrightarrow{\mathrm{x}}^{1}, \ldots, \overrightarrow{\mathrm{x}}^{1}\right)\right) .
\end{aligned}
$$


For any given interpretation $\mathcal{I}$, we build the corresponding interpretation $\mathcal{I}^{\prime}$ in two steps. In the first step, we map the interpretation $\mathcal{I}$ into an interpretation $\pi(\mathcal{I})$ (projection on the starting point) defined as follows.

Definition 3.5 Let $\mathcal{I}$ be an interpretation for $\mathcal{L}_{M}^{2}$ satisfying the formula

$$
\psi=\bigwedge_{\mathrm{h}=1}^{\mathrm{l}} \xi\left(\mathrm{x}_{1}^{\mathrm{h}}, \ldots, \mathrm{x}_{\mathrm{n}}^{\mathrm{h}}, \mathrm{x}^{\mathrm{h}}\right) \wedge \tau_{1}\left(\varphi\left(\overrightarrow{\mathrm{x}}^{1}, \ldots, \overrightarrow{\mathrm{x}}^{1}\right)\right) .
$$

This implies that, for $1 \leq h \leq l$, there exists $i$ such that either $1 \leq i<n, \mathcal{I}\left(\mathrm{x}_{\mathrm{i}}^{\mathrm{h}}\right) \leq \mathcal{I}\left(\mathrm{x}^{\mathrm{h}}\right)$ and $\mathcal{I}\left(\mathrm{x}_{\mathrm{i}+1}^{\mathrm{h}}\right)>\mathcal{I}\left(\mathrm{x}^{\mathrm{h}}\right)$, or $i=n$ and $\mathcal{I}\left(\mathrm{x}^{\mathrm{h}}\right)=\mathcal{I}\left(\mathrm{x}_{\mathrm{i}}^{\mathrm{h}}\right)$. The interpretation $\pi(\mathcal{I})$ for $\psi$ assigns to the free individual variables of $\psi$ the same values as $\mathcal{I}$, and for $j=1, \ldots i$ and $k=1, \ldots, m, \pi(\mathcal{I})\left(\mathrm{x}_{j}^{\mathrm{h}}\right) \in p_{k, j}^{\pi(\mathcal{I})}$ if and only if $\mathcal{I}\left(\mathrm{x}^{\mathrm{h}}\right) \in p_{k, j}^{\mathcal{J}}$.

For all the other elements $x$ of the domain (including $\left.\mathcal{I}\left(\mathrm{x}^{1}\right), \ldots \mathcal{I}\left(\mathrm{x}^{1}\right)\right), x \in p_{k, i}^{\pi(\mathcal{I})}$ if and only if $x \in p_{k, i}^{\mathfrak{g}}$, for $k=1, \ldots, m$ and $i=1, \ldots, n$.

Lemma 3.6 For each formula $\bigwedge_{\mathrm{h}=1}^{\mathrm{l}} \xi\left(\mathrm{x}_{1}^{\mathrm{h}}, \ldots, \mathrm{x}_{\mathrm{n}}^{\mathrm{h}}, \mathrm{x}^{\mathrm{h}}\right) \wedge \tau_{1}\left(\varphi\left(\overrightarrow{\mathrm{x}}^{1}, \ldots, \overrightarrow{\mathrm{x}}^{1}\right)\right)$ and each interpretation $\mathcal{I}$,

$$
\begin{aligned}
\mathcal{I} \models & \bigwedge_{\mathrm{h}=1}^{1} \xi\left(\mathrm{x}_{1}^{\mathrm{h}}, \ldots, \mathrm{x}_{\mathrm{n}}^{\mathrm{h}}, \mathrm{x}^{\mathrm{h}}\right) \wedge \tau_{1}\left(\varphi\left(\overrightarrow{\mathrm{x}}^{1}, \ldots, \overrightarrow{\mathrm{x}}^{1}\right)\right) \text { iff } \\
& \pi(\mathcal{J}) \models \bigwedge_{\mathrm{h}=1}^{1} \xi\left(\mathrm{x}_{1}^{\mathrm{h}}, \ldots, \mathrm{x}_{\mathrm{n}}^{\mathrm{h}}, \mathrm{x}^{\mathrm{h}}\right) \wedge \tau_{1}\left(\varphi\left(\overrightarrow{\mathrm{x}}^{1}, \ldots, \overrightarrow{\mathrm{x}}^{1}\right)\right) .
\end{aligned}
$$

The proof is straightforward and is left to the reader.

To obtain the desired interpretation, each $\pi$-interpretation $\mathcal{I}$ is then mapped into an interpretation $\epsilon(\mathcal{I})$ (expansion over the whole interval) defined as follows.

Definition 3.7 Let $\mathcal{I}$ be an interpretation for $\mathcal{L}_{M}^{2}$ satisfying the formula

$$
\psi=\bigwedge_{\mathrm{h}=1}^{\mathrm{l}} \xi\left(\mathrm{x}_{1}^{\mathrm{h}}, \ldots, \mathrm{x}_{\mathrm{n}}^{\mathrm{h}}, \mathrm{x}^{\mathrm{h}}\right) \wedge \tau_{1}\left(\varphi\left(\overrightarrow{\mathrm{x}}^{1}, \ldots, \overrightarrow{\mathrm{x}}^{1}\right)\right) .
$$

The interpretation $\epsilon(\mathcal{g})$ for $\psi$ assigns to the free individual variables of $\psi$ the same values as $\mathcal{I}$ and for $k=1, \ldots, m$ and $i=1, \ldots, n$,

$$
p_{k, i}^{\epsilon(\mathcal{J})}=\left\{x \mid\left\lfloor x / c f_{i, n}\right\rfloor \cdot c f_{i, n} \in p_{k, i}^{\mathcal{I}}\right\} .
$$

$\epsilon(\mathcal{J})$ is the interpretation that, for every $x \in\left[q \cdot c f_{i, n},(q+1) \cdot c f_{i, n}\right)$, sets the truth value of $p_{k, i}$ on $x$ equal to the truth value of $p_{k, i}$ on $q \cdot c f_{i, n}$.

The following lemma holds.

Lemma 3.8 For each formula $\bigwedge_{\mathrm{h}=1}^{\mathrm{l}} \xi\left(\mathrm{x}_{1}^{\mathrm{h}}, \ldots, \mathrm{x}_{\mathrm{n}}^{\mathrm{h}}, \mathrm{x}^{\mathrm{h}}\right) \wedge \tau_{1}\left(\varphi\left(\overrightarrow{\mathrm{x}}^{1}, \ldots, \overrightarrow{\mathrm{x}}^{1}\right)\right)$ and each interpretation $\mathcal{I}$ for it,

$$
\begin{aligned}
\pi(\mathcal{J}) & \models \bigwedge_{\mathrm{h}=1}^{1} \xi\left(\mathrm{x}_{1}^{\mathrm{h}}, \ldots, \mathrm{x}_{\mathrm{n}}^{\mathrm{h}}, \mathrm{x}^{\mathrm{h}}\right) \wedge \tau_{1}\left(\varphi\left(\overrightarrow{\mathrm{x}}^{1}, \ldots, \overrightarrow{\mathrm{x}}^{1}\right)\right) \text { iff } \\
\epsilon(\pi(\mathcal{J})) & \models \bigwedge_{\mathrm{h}=1}^{1} \xi\left(\mathrm{x}_{1}^{\mathrm{h}}, \ldots, \mathrm{x}_{\mathrm{n}}^{\mathrm{h}}, \mathrm{x}^{\mathrm{h}}\right) \wedge \tau_{1}\left(\varphi\left(\overrightarrow{\mathrm{x}}^{1}, \ldots, \overrightarrow{\mathrm{x}}^{1}\right)\right) .
\end{aligned}
$$


Moreover, there exists an interpretation I for $\varphi\left(\vec{x}^{1}, \ldots, \vec{x}^{l}\right) \in \mathcal{L}_{L M}^{2}$ such that $\tau_{1}(I)=$ $\epsilon(\pi(\mathcal{I}))$.

Proof: The formulas we are interested in only constrain the truth values of predicates at $x^{1}, \ldots, x^{l}$. On the other hand, from the definition of $\epsilon$ we have that, for every $p_{k, i}$, the truth-value of $\epsilon(\pi(\mathcal{I}))$ and $\pi(\mathcal{I})$ at $x^{1}, \ldots, x^{l}$ is the same, since $\pi(\mathcal{J})$ assigns the same truth values at $x^{1}, \ldots, x^{l}$ and $\left\lfloor x^{1} / c f_{i, n}\right\rfloor \cdot c f_{i, n}, \ldots,\left\lfloor x^{l} / c f_{i, n}\right\rfloor \cdot c f_{i, n}$, respectively. Therefore, the thesis follows from the definitions of $\pi$ and $\epsilon$.

Furthermore, let $I$ an interpretation for $\varphi\left(\overrightarrow{\mathrm{x}}^{1}, \ldots, \overrightarrow{\mathrm{x}}^{1}\right) \in \mathcal{L}_{L M}^{2}$ such that:

$$
p_{k, i}^{I}=\left\{x \mid x \cdot c f_{i, n} \in p_{k, i}^{\epsilon(\pi(\mathcal{I}))}\right\} .
$$

It follows that $\tau_{1}(I)=\epsilon(\pi(\mathcal{J}))$.

Notice that every $\epsilon$-interpretation is a $\tau_{1}(I)$ interpretation, for some interpretation $I$ for $\mathcal{L}_{L M}^{2}$.

Now, our main preservation result follows from the previous lemmas.

Theorem 3.9 For every sentence $\varphi$ of $\mathcal{L}_{L M}^{2}$, with free predicate symbols $p_{1}, \ldots, p_{m}$, there exists a sentence $\psi\left(=\tau_{1}(\varphi)\right)$ of $\mathcal{L}^{M}$, with free predicate symbols $p_{1,1}, \ldots, p_{m, n}$, such that $\varphi$ is valid (satisfiable) in $T_{L M}$ if and only if $\psi$ is valid (satisfiable) in $T_{M}$. Furthermore, if $\varphi \in \mathcal{L}_{L M}$, then $\psi \in \mathcal{L}_{M}$.

Proof: On the one hand, from Lemma 3.4. it follows that, for any sentence $\varphi \in \mathcal{L}_{L M}^{2}$, if $\varphi$ is satisfiable, then $\tau_{1}(\varphi) \in \mathcal{L}_{M}^{2}$ is satisfiable, and, conversely, if $\tau_{1}(\varphi)$ is valid, then $\varphi$ is valid. On the other hand, Lemmas 3.6 and 3.8 rove that if $\varphi$ is valid, then $\tau_{1}(\varphi)$ is valid, and, conversely, if $\tau_{1}(\varphi)$ is satisfiable, then $\varphi$ is satisfiable.

3.3 Coding metric information The second step of the translation is the mapping of $\mathcal{L}_{M}^{2}$ formulas into $\mathcal{L}^{2}$ ones. It is performed by a function $\tau_{2}$ that reduces each formula $\psi \in \mathcal{L}_{M}^{2}$ to a formula $\tau_{2}(\psi) \in \mathcal{L}^{2}$ devoid of occurrences of the successor function and of congruence predicates. Moreover, $\tau_{2}$ does not change the set of free individual variables of $\varphi$, so that if $\varphi$ does not contain any free individual variable, no free individual variables occur in $\tau_{2}(\varphi)$.

Before entering into the details of the definition of $\tau_{2}$, we point out that at this stage we could simply use the same technique employed in 10 to map $\mathcal{L}_{M}^{2}$ formulas into $\mathcal{L}^{2}$ formulas. Even if the theory of metric temporal structures $T_{M}$ does not support an explicit notion of state distinct from time ${ }^{5}$, it can be easily reformulated in terms of a particular two-sorted second-order theory of timed state sequences whose time function is the identity function. Nevertheless, we will introduce and briefly discuss $\tau_{2}$, mainly because it turns out to be a (rather elegant and) essentially compositional translation for our setting.

Terms of $\mathcal{L}_{M}^{2}$ are defined as follows: (i) the zero constant 0 is a term; (ii) each variable $\mathrm{x}$ is a term; (iii) if $\mathrm{t}$ is a term, then $\mathrm{t}+1$ is a term; (iv) nothing else is a term. In the following, we will use $+\mathrm{n}$ as a shorthand for $n$ superpositions of +1 . As in the case of $\mathcal{L}_{L M}^{2}$-formulas, we assume without loss of generality that terms appearing in atomic formulas of $\mathcal{L}_{M}^{2}$ which are not equalities are variables. Atomic formulas are of the forms $t_{1}=t_{2}, x \leq y, x \equiv_{d} y$, and $p_{k}(t)$, where $t_{1}, t_{2}$ are terms, $x, y$ are variables, $\leq$ is the binary ordering relation, $\equiv_{\mathrm{d}}$ is a binary congruence relation, and 
$\mathrm{p}_{\mathrm{k}}$ is an uninterpreted unary predicate symbol. Compound $\mathcal{L}_{M}^{2}$-formulas can be obtained by means of logical connectives and quantifications over individual and predicate variables. In particular, inequalities $(\neq)$ and strict inequalities $(<)$ can be defined in terms of $=$ and $\leq$ in the usual way.

With regard to compound $\mathcal{L}_{M}^{2}$-formulas, $\tau_{2}$ distributes over quantifiers, negation, and conjunction. Therefore, we only need to define $\tau_{2}$ on atomic formulas. We first consider atomic $\mathcal{L}_{M}^{2}$-formulas of the form $\mathrm{y}=\mathrm{x}+\mathrm{n}$. We will show that they can be reduced to $\mathcal{L}^{2}$-formulas involving first-order quantification over $n+1$ time variables and devoid of any occurrence of +1 . Moreover, on the basis of the definition of the successor function, it is straightforward to prove that formulas of the form $\mathrm{x}+\mathrm{n}=$ $\mathrm{y}+\mathrm{m}$, with $m, n>0$, can be reduced either to formulas of the form $\mathrm{x}=\mathrm{y}+\mathrm{m}^{\prime}$ or to formulas of the form $\mathrm{x}+\mathrm{n}^{\prime}=\mathrm{y}$, with $m^{\prime}, n^{\prime} \geq 0$ and $\mathrm{x}+0$ to be read as $\mathrm{x}$. Let us start with the case $n=1$. Let $\psi$ be the $\mathcal{L}_{M}^{2}$-formula $\mathrm{y}=\mathrm{x}+1$. The translation function $\tau_{2}$ transforms it into an equivalent formula devoid of occurrences of +1 :

$$
\tau_{2}(\mathrm{y}=\mathrm{x}+1)=\exists \mathrm{x}_{1}\left(\mathrm{x}<\mathrm{x}_{1} \wedge \mathrm{y}=\mathrm{x}_{1} \wedge \forall \overline{\mathrm{x}}\left(\mathrm{x} \leq \overline{\mathrm{x}} \leq \mathrm{x}_{1} \rightarrow\left(\overline{\mathrm{x}}=\mathrm{x} \vee \overline{\mathrm{x}}=\mathrm{x}_{1}\right)\right)\right) .
$$

It is easy to generalize this transformation to any $\mathcal{L}_{M}^{2}$-formula y $=\mathrm{x}+\mathrm{n}$, with $n>1$ :

$$
\begin{aligned}
\tau_{2}(\mathrm{y}=\mathrm{x}+\mathrm{n})= & \exists \mathrm{x}_{1}, \ldots, \mathrm{x}_{\mathrm{n}}\left(\mathrm{x}<\mathrm{x}_{1}<\ldots<\mathrm{x}_{\mathrm{n}} \wedge \mathrm{y}=\mathrm{x}_{\mathrm{n}} \wedge\right. \\
& \left.\wedge \forall \overline{\mathrm{x}}\left(\mathrm{x} \leq \overline{\mathrm{x}} \leq \mathrm{x}_{\mathrm{n}} \rightarrow\left(\overline{\mathrm{x}}=\mathrm{x} \vee \ldots \vee \overline{\mathrm{x}}=\mathrm{x}_{\mathrm{n}}\right)\right)\right) .
\end{aligned}
$$

The case of atomic $\mathcal{L}_{M}^{2}$-formulas of the form $\mathrm{y}=\mathrm{n}$, where $\mathrm{n}$ stands for $0+\mathrm{n}$, is analogous, and thus omitted. Equalities of the form $\mathrm{x}=\mathrm{y}$ as well as atomic formulas of the form $\mathrm{x} \leq \mathrm{y}$ and $\mathrm{p}_{\mathrm{k}}(\mathrm{x})$ are left unchanged. Let us consider now atomic formulas of the form $\mathrm{x} \equiv_{\mathrm{d}} \mathrm{y}$. Each binary congruence relation $\equiv_{\mathrm{d}}$ partitions the set of time instants into $d$ disjoint classes. For each class of time instants which are congruent modulo $d$ with $i$, with $0 \leq i \leq d-1, \tau_{2}$ introduces a monadic predicate of the form Tcong $_{\mathrm{d}, \mathrm{i}}$. It is defined as follows:

$$
\tau_{2}\left(\mathrm{x} \equiv_{\mathrm{d}} \mathrm{y}\right)=\bigwedge_{\mathrm{i}=0}^{\mathrm{d}-1}\left(\operatorname{Tcong}_{\mathrm{d}, \mathrm{i}}(\mathrm{x}) \leftrightarrow \operatorname{Tcong}_{\mathrm{d}, \mathrm{i}}(\mathrm{y})\right)
$$

where $\bigwedge_{i=0}^{d-1}$ denotes the usual shorthand. Since for every congruence relation $\equiv_{d}$ the corresponding predicates $T \operatorname{cong}_{\mathrm{d}, 0}, \ldots, T \operatorname{con}_{\mathrm{d}, \mathrm{d}-1}$ are uninterpreted monadic predicate symbols, the following conditions must be added:

(a) for each congruence relation $\equiv_{d}($ in $\psi), T c o n g_{\mathrm{d}, 0}$ holds at time instant 0 (in $\left.\tau_{2}(\psi)\right)$

(b) for each congruence relation $\equiv_{d}$, and each time instant $x$, there exists one and only one index $i$, with $0 \leq i \leq d-1$, such that $T$ cong $g_{\mathrm{d}, \mathrm{i}}$ holds at $x$;

(c) for each congruence relation $\equiv_{d}$, each index $i$, with $0 \leq i \leq d-1$, and each time instant $x$, if $T \operatorname{cong}_{\mathrm{d}, \mathrm{i}}$ holds at time instant $x$, then $T \operatorname{cong}_{\mathrm{d}, \mathrm{i}+1 \operatorname{modd}}$ holds at time instant $x+1$.

Condition (a) links time-congruence predicates corresponding to different congruence relations (it provides a sort of initial synchronization); (b) and (c) link timecongruence predicates corresponding to the same congruence relation. Formally, for 
each congruence relation $\equiv_{\mathrm{d}}$ in $\psi$, let $\chi\left(T \operatorname{con} g_{\mathrm{d}, 0}, \ldots, \operatorname{Tcong}_{\mathrm{d}, \mathrm{d}-1}\right)$ be the formula:

$$
\begin{aligned}
& \operatorname{Tcong}_{d, 0}(0) \wedge \forall x\left(\bigvee_{i=0}^{d-1}\left(T \operatorname{cong}_{d, i}(x) \wedge \bigwedge_{j \neq i} \neg \operatorname{Tcong}_{d, j}(x)\right) \wedge\right. \\
&\left.\wedge \bigwedge_{i=0}^{d-1}\left(\operatorname{Tcong}_{d, i}(x) \rightarrow \operatorname{Tcong}_{d, i+1 \text { modd }}(x+1)\right)\right)
\end{aligned}
$$

where the usual shorthands have been used.

The translation of $\mathcal{L}_{M}^{2}$-formulas $\psi$ is thus defined by adding, for each distinct congruence relation $\equiv_{\mathrm{d}}$ occurring in $\psi$, the corresponding conjunct $\chi\left(T \operatorname{con}_{\mathrm{d}, 0}, \ldots\right.$, $T$ cong $\left._{d, d-1}\right)$. The resulting formula belongs to $\mathcal{L}^{2}$. Therefore, in order to prove that the validity (satisfiability) problem for $\mathcal{L}_{M}^{2}$ is decidable, we only need to show that a sentence $\psi$ is valid (satisfiable) in $T_{M}$ if and only if $\tau_{2}(\psi)$ is valid (satisfiable) in S1S.

Theorem 3.10 For every formula $\psi$ of $\mathcal{L}_{M}^{2}$, there exists a formula $\theta$ of $\mathcal{L}^{2}$, which contains the additional time-congruence predicates $\mathrm{Tcong}_{\mathrm{d}_{1}, 0}, \ldots, \mathrm{Tcong}_{\mathrm{d}_{1}, \mathrm{~d}_{1}-1}, \ldots$, Tcong $_{\mathrm{d}_{1}, 0}, \ldots$, Tcong $_{\mathrm{d}_{1}, \mathrm{~d}_{1}-1}$, such that $\psi$ is valid (satisfiable) in $T_{M}$ if and only if $\theta$ is valid (satisfiable) in S1S. Furthermore, if $\psi \in \mathcal{L}_{M}$, then $\theta \in \mathcal{L}$.

Take $\tau_{2}(\psi)$ as $\theta$. The proof is similar to the one given in [1], and thus omitted.

On the basis of Theorems 3.9 and 3.10. we can conclude that the following holds:

Theorem 3.11 For every formula $\varphi$ of $\mathcal{L}_{L M}^{2}$, there exists a formula $\theta\left(i . e ., \tau_{2}\left(\tau_{1}(\varphi)\right)\right.$ ) of $\mathcal{L}^{2}$ such that $\varphi$ is valid (satisfiable) in $T_{L M}$ if and only if $\theta$ is valid (satisfiable) in S1S. Furthermore, if $\varphi \in \mathcal{L}_{M}$, then $\theta \in \mathcal{L}$.

Hence, from the the decidability of S1S, the decidability of $T_{L M}$ follows:

Corollary 3.12 The theory of finitely-layered metric temporal structures is decidable.

Remark 3.13 The above result can be applied to obtain decidability results for semantically defined metric and layered temporal logics. Indeed, we first identified a relevant class of temporal structures, namely, the class of finitely-layered metric temporal structures; then, we defined the corresponding theory $T_{L M}$ and showed that such a theory can be reduced to S1S. An axiomatic counterpart of $T_{L M}$ can be obtained extending a simplified variant of TPTL (real-time propositional temporal logic), where state variables are replaced by time variables and $\bigcirc$ is interpreted as the successor over time, with contextual and projection operators of $M L T L$.

4 Conclusions and further work In this paper we have proved the decidability of the theory of finitely-layered metric temporal structures through its reduction to the decidable theory S1S. Since the validity problem is non-elementary already for the classical first-order theory of natural numbers with linear order and monadic predicates, it is obviously non-elementary also for the considered theory. Nevertheless, we expect that modal counterparts of the proposed theory corresponding to an elementary, yet expressively complete, fragment of $\mathcal{L}_{L M}^{2}$ can be identified. To this end, we are currently analyzing suitable propositional fragments of MLTL. We are also 
considering the problem of executing logical specifications. In principle, decidability proof methods outline an effective procedure to prove the satisfiability and/or validity of a formula. However, as soon as certain assumptions about the nature of the temporal domain and the available set of operations are relaxed, the satisfiability/validity problem becomes (strongly) undecidable. In this respect, proof-theoretic approaches seem to offer a valid alternative, e.g., D’ Agostino et al. in [5]. Finally, in 16 we extended the decidability results given in this paper to $\omega$-layered metric temporal structures consisting of a denumerable set of (either arbitrarily coarse or arbitrarily fine) temporal domains.

Acknowledgments This work has been supported by funds MURST $40 \%$ and $60 \%$. The first author was supported by a grant from the Italian Consiglio Nazionale delle Ricerche (CNR). This work was carried out while the first author was visiting ILLC, University of Amsterdam. We would like to thank Maarten de Rijke for many useful discussions and inspiring comments.

\section{NOTES}

1. More precisely, the problem of checking the validity of a formula $\mathcal{F}$ can be reduced to the decidable problem of checking whether or not the language recognized by the Büchi automaton corresponding to $\neg \mathcal{F}$ is empty, whereas the problem of checking the satisfiability of a formula $\mathcal{F}$ can be reduced to the decidable problem of checking whether or not the language recognized by the Büchi automaton corresponding to $\mathcal{F}$ is not empty.

2. The original proof is given in 11. Corrections and remarks on this proof can be found in Montanari and Policriti 15].

3. The addition of the consistency rule would make such a splitting of $p_{\mathrm{k}}$ unnecessary.

4. Notice that, if the $\mathcal{L}_{L M}^{2}$-formula $\varphi$ is a sentence, the corresponding $\mathcal{L}_{M}^{2}$-formula is the sentence $\tau_{1}(\varphi)$ (no free variables occur in $\varphi$ ).

5. It is worth noting that a differentiation between the notions of state and time can be recovered using granularity. Upward projection can indeed map two time instants which are distinct with respect to the domain they finely belong to into the same time instant of a coarser domain. With respect to the coarser domain, the original time instants can be viewed as an ordered pair of simultaneous states.

\section{REFERENCES}

[1] Alur, R., and T. A. Henzinger, "Real-time logics: complexity and expressiveness," Information and Computation, vol. 104 (1993), pp. 35-77. Zbl 0791.68103 MR 94c:03036 1.3.1.3.1.3.1.13.3.3.3.4

[2] Büchi, J. R., "Weak second-order arithmetic and finite automata," Zeitschrift für mathematishce Logik und Grundlagen der Mathematik, vol. 6 (1960), pp. 66-92. MR 23:A2317 3.1

[3] Büchi, J. R., "On a decision method in restricted second-order arithmetic," pp. 1-11 in Proceedings of 1st International Congress on Logic, Methodology, and Philosophy of Science edited by E. Nagel, P. Suppes, and A. Tarski, Stanford University Press, Stanford, 1962. MR 32:1116 3.1 
[4] Ciapessoni, E., E. Corsetti, A. Montanari, and P. San Pietro, "Embedding time granularity in a logical specification language for synchronous real-time systems." Science of Computer Programming, vol. 20 (1993), pp. 141-171. Zbl 0781.68083MR 94j:68196 1,11

[5] D'Agostino, G., A. Montanari, and A. Policriti, "A set-theoretic translation method for polymodal logics," Journal of Automated Reasoning, vol. 15 (1995), pp. 317-337. Zb] 0840.03015 MR 96i:03024 4

[6] Ershov, Y. L., I. A. Lavrov, A. D. Taimanov, and M. A. Taitslin, "Elementary theories," Russian Mathematical Surveys, vol. 20 (1965), pp. 35-105.Zbl 0199.03001 1

[7] Euzenat, J., "An algebraic approach for granularity in qualitative space and time representation," pp. 894-900 in Proceedings of IJCAI '95, Morgan Kaufmann, San Mateo, 1995.

[8] Fiadeiro, J. and T. Maibaum, "Sometimes 'Tomorrow' is 'Sometimes'-action refinement in a temporal logic of objects," pp. 48-66 in Proceedings of ICTL '94, LNAI 827, Springer-Verlag, Berlin, 1992. 1]

[9] Finger, M., and D. Gabbay, "Combining temporal logic systems," Notre Dame Journal of Formal Logic, vol. 37 (1996), pp. 204-232. Zbl 0857.03008 MR 97d:03011 1

[10] Gabbay, D., A. Pnueli, S. Shelah, and J. Stavi, "On the temporal analysis of fairness," pp. 163-173 in Proceedings of the 7th Annual Symposium on Principles of Programming Languages, ACM Press, New York, 1980.

[11] Koymans, R., "Specifying message passing and time-critical systems with temporal logic," LNCS 651, Springer-Verlag, Berlin, 1992.Zbl 0806.68074|MR 94c:68138 1

[12] Manna, Z., and A. Pnueli, The Temporal Logic of Reactive and Concurrent Systems, Springer-Verlag, Berlin, 1992.Zbl 0753.68003|MR 93e:68073 3.1

[13] Montanari, A., "A metric and layered temporal logic for time granularity, synchrony and asynchrony," pp. 49-58 in Proceedings of the ICTL '94 Workshop, MPI-I-94-230, edited by H. J. Ohlbach, Max-Planck-Institut für Informatik, Saarbruecken,1994.

[14] Montanari, A., and M. de Rijke, "Completeness results for two-sorted metric temporal logics," pp. 385-399 in Proceedings of the AMAST '95, LNCS 936, Springer-Verlag, Berlin, 1995.

[15] Montanari, A., and A. Policriti, "A decidable theory of finitely-layered metric temporal structures,” Report ML-96-06, ILLC, University of Amsterdam, July, 1996. 4

[16] Montanari, A., A. Peron, and A. Policriti, "Decidabable theories of $\omega$-layered metric temporal structures," Report ML-96-07, ILLC, University of Amsterdam, July, 1996. 4

[17] Rabin, M. O., "Decidable theories," pp. 595-629 in Handbook of Mathematical Logic, edited by J. Barwise, North-Holland, Ansterdam, 1977. Zbl 0443.03001 MR 56:15351

[18] Thomas, W., "Automata on infinite objects," pp. 131-191 in Handbook of Theoretical Computer Science, edited by J. van Leeuwen, Elsevier, Amsterdam, 1990. Zbl 0900.68316|MR 1127189 1

[19] van Benthem J., "Temporal logic," pp. 241-350 in Handbook of Logic in Artificial Intelligence and Logic Programming, Vol. 4, edited by D. Gabbay, C. Hogger, and J. Robinson, Oxford University Press, Oxford,1995. MR 97a:03023 
[20] Wang, X., C. Bettini, A. Brodsky, and S. Jajodia, "Logical design for temporal databases with multiple granularities," forthcoming in ACM TODS. 1

Dipartimento di Matematica e Informatica

Università di Udine

Via delle Scienze, 206 - 33100 Udine, Italy.

email: montana@dimi.uniud.it

email: policrit@dimi.uniud.it 